\title{
Codes from adjacency matrices of uniform subset graphs
}

\author{
W. Fish • J.D. Key • E. Mwambene
}

Received: date / Accepted: date

\begin{abstract}
Studies of the $p$-ary codes from the adjacency matrices of uniform subset graphs $\Gamma(n, k, r)$ and their reflexive associates have shown that a particular family of codes defined on the subsets are intimately related to the codes from these graphs. We describe these codes here and examine their relation to some particular classes of uniform subset graphs. In particular we include a complete analysis of the $p$-ary codes from $\Gamma(n, 3, r)$ for $p \geq 5$, thus extending earlier results for $p=2,3$.
\end{abstract}

Keywords Uniform subset graphs · Codes from graphs

Mathematics Subject Classification (2000) 05C50 - 94B05

\section{Introduction}

Studies of codes obtained from the row span over finite fields of adjacency matrices of uniform subset graphs $\Gamma(n, k, r)$ and their reflexive counterparts $R \Gamma(n, k, r)$, for example in $[9,14,15,12,11]$, have shown that a class of codes, the members of which are denoted by $W_{i}$, for $0 \leq i \leq k$, and another, $W_{\Pi}$, are present in the ambient space $\mathbb{F}_{p}^{V}$ of these codes (where $V=\Omega^{\{k\}}$, the set of subsets of size $k$ of a set $\Omega$ of size $n$, and $p$ is a prime), and closely related to the specific codes from the graphs. Since codes from adjacency matrices of graphs have not been easy to classify, a uniform approach for at least one class of graphs would seem to be desirable.

In this paper we define these $W$ codes for general uniform subset graphs, and obtain some general properties, before we illustrate their use in the $p$-ary

Fish, Key, Mwambene

Department of Mathematics and Applied Mathematics

University of the Western Cape

7535 Bellville, South Africa

E-mail: wfish@uwc.ac.za, keyj@clemson.edu, emwambene@uwc.ac.za 
codes of the specific case of $\Gamma(n, 3, r)$ and $R \Gamma(n, 3, r)$, for $p \geq 5$, since the cases $p=2,3$ have already been established in $[9,14,15,12,11]$.

After a general background section, we define the $W$ codes in Section 3, Equations (1), (2), (3), and obtain some further general results about these codes in Sections 4, 5. We then consider their relationship to the codes from the graphs $\Gamma(n, k, r)$ in Section 6 , and in particular for $k=3$ in Section 7 . Our conclusions for $k=3$, for all primes $p$, are shown in tables in Sections 8.1 to 8.7. Any computations were done with Magma [3,5].

\section{Terminology and background}

The notation for designs and codes is as in [2]. An incidence structure $\mathcal{D}=$ $(\mathcal{P}, \mathcal{B}, \mathcal{J})$, with point set $\mathcal{P}$, block set $\mathcal{B}$ and incidence $\mathcal{J}$ is a $t-(v, k, \lambda)$ design if $|\mathcal{P}|=v$, every block $B \in \mathcal{B}$ is incident with precisely $k$ points, and every $t$ distinct points are together incident with precisely $\lambda$ blocks. The code $C_{F}(\mathcal{D})$ of the design $\mathcal{D}$ over the finite field $F$ is the space spanned by the incidence vectors of the blocks over $F$. If $\mathcal{Q}$ is any subset of $\mathcal{P}$, then we will denote the incidence vector of $\mathcal{Q}$ by $\boldsymbol{v}^{\mathcal{Q}}$, and if $\mathcal{Q}=\{P\}$ where $P \in \mathcal{P}$, then we will write $v^{P}$ instead of $v^{\{P\}}$. Thus $C_{F}(\mathcal{D})=\left\langle v^{B} \mid B \in \mathcal{B}\right\rangle$, and is a subspace of $F^{\mathcal{P}}$, the full vector space of functions from $\mathcal{P}$ to $F$. For any $w \in F^{\mathcal{P}}$ and $P \in \mathcal{P}, \boldsymbol{w}(\boldsymbol{P})$ denotes the value of $w$ at $P$. If $F=\mathbb{F}_{p}$ then the $\boldsymbol{p}$-rank of the design, written $\operatorname{rank}_{p}(\mathcal{D})$, is the dimension of its code $C_{F}(\mathcal{D})$; for $F=\mathbb{F}_{p}$ we usually write $C_{p}(\mathcal{D})$ for $C_{F}(\mathcal{D})$.

All the codes here are linear codes, and the notation $[n, k, d]_{q}$ will be used for a $q$-ary code $C$ of length $n$, dimension $k$, and minimum weight $d$, where the weight $\operatorname{wt}(\boldsymbol{v})$ of a vector $v$ is the number of non-zero coordinate entries. Vectors in a code are also called words. The support, $\operatorname{Supp}(v)$, of a vector $v$ is the set of coordinate positions where the entry in $v$ is non-zero. So $|\operatorname{Supp}(v)|=\operatorname{wt}(v)$. The dual code $C^{\perp}$ is the orthogonal under the standard inner product (,), i.e. $C^{\perp}=\left\{v \in F^{n} \mid(v, c)=0\right.$ for all $\left.c \in C\right\}$. The hull of a code $C$ is the self-orthogonal code $C \cap C^{\perp}$. Following [19], a linear code $C$ is $L C D$ (linear with complementary dual) if $\operatorname{Hull}(C)=\{0\}$. The all-one vector will be denoted by $\boldsymbol{\jmath}$, and is the vector with all entries equal to 1 . If we need to specify the length $\mathbf{m}$ of the all-one vector, we write $\boldsymbol{J}_{\mathbf{m}}$. A constant vector is a non-zero vector in which all the non-zero entries are the same. We call two linear codes isomorphic (or permutation isomorphic) if they can be obtained from one another by permuting the coordinate positions. An automorphism of a code $C$ is an isomorphism from $C$ to $C$. The automorphism group will be denoted by $\operatorname{Aut}(C)$, also called the permutation group of $C$, and denoted by $\operatorname{PAut}(C)$ in [13].

The graphs, $\Gamma=(V, E)$ with vertex set $V$ and edge set $E$, discussed here are undirected with no loops, apart from the case where all loops are included, in which case the graph is called reflexive. The reflexive graph from $\Gamma$ is denoted by $R \Gamma$. If $x, y \in V$ and $x$ and $y$ are adjacent, we write $x \sim y$, and $x y$ or $[x, y]$ for the edge in $E$ that they define. The set of neighbours 
of $x \in V$ is denoted by $N(x)$, and the valency of $x$ is $|N(x)| . \Gamma$ is regular if all the vertices have the same valency.

An adjacency matrix $A$ is a $|V| \times|V|$ symmetric matrix with entries $a_{i j}$ such that $a_{i j}=1$ if vertices $x_{i}$ and $x_{j}$ are adjacent, and $a_{i j}=0$ otherwise. If $\Gamma=(V, E)$ is a graph with adjacency matrix $A$ then $A+I_{|V|}$ is an adjacency matrix for the reflexive graph $R \Gamma$ from $\Gamma$.

The code of $\Gamma=(V, E)$ over a finite field $F$ is the row span of an adjacency matrix $A$ over the field $F$, denoted by $C_{F}(\Gamma)$ or $C_{F}(A)$. Similarly for $R \Gamma$. The dimension of the code is the rank of the matrix over $F$, also written $\operatorname{rank}_{p}(A)$ if $F=\mathbb{F}_{p}$, in which case we will speak of the $p$-rank of $A$ or $\Gamma$ (respectively $R \Gamma$ ), and write $C_{p}(\Gamma)$ or $C_{p}(A)$ for the code (respectively $C_{p}(R \Gamma)$ or $C_{p}\left(A+I_{|V|}\right)$ ). The ambient space of these codes is $\mathbb{F}_{p}^{V}$.

The uniform subset graph $\Gamma(n, k, r)$ has for vertices the set of all subsets of size $k$ of a set of size $n$ with two $k$-subsets $x$ and $y$ defined to be adjacent if $|x \cap y|=r$. Then $\Gamma(n, k, r)$ is regular of valency $\left(\begin{array}{l}k \\ r\end{array}\right)\left(\begin{array}{l}n-k \\ k-r\end{array}\right)$. The symmetric group $S_{n}$ always acts on $\Gamma(n, k, r)$, transitively on vertices and edges. Similarly for $R \Gamma(n, k, r)$. We take $n \geq 2 k$, and generally $n \geq 2 k+1$.

\section{The $W$ codes}

We define here the $W$ codes as mentioned, and develop a few of their general properties, but we will restrict mainly to $k=3$ and mostly leave the further more general study for $k \geq 4$ to a future paper.

Let $\Omega=\{1, \ldots, n\}, k \leq \frac{n}{2}, V=\Omega^{\{k\}}$. For $0 \leq s \leq n$ let $\Omega^{\{s\}}$ denote the set of $s$-subsets of $\Omega$. All spans are over $\mathbb{F}_{p}$ for a given prime $p$, and all the codes are subcodes of $\mathbb{F}_{p}^{V}$, where we fix $n, k$.

For $\Lambda \in \Omega^{\{s\}}$ and $0 \leq s \leq k$, define in $\mathbb{F}_{p}^{V}$,

$w_{\Lambda}=\sum_{\substack{\Lambda_{1} \in \Omega\{k-s\} \\ \Lambda \cap \Lambda_{1}=\emptyset}} v^{\Lambda \cup \Lambda_{1}}, W_{s}=\left\langle w_{\Lambda} \mid \Lambda \in \Omega^{\{s\}}\right\rangle, E\left(W_{s}\right)=\left\langle w_{\Lambda_{1}}-w_{\Lambda_{2}} \mid \Lambda_{i} \in \Omega^{\{s\}}\right\rangle$.

Identities 1 For $\Lambda \in \Omega^{\{s\}}$ and $0 \leq s \leq k$,

(1) $w_{\emptyset}=\boldsymbol{\jmath}$; (2) $\operatorname{wt}\left(w_{\Lambda}\right)=\left(\begin{array}{l}n-s \\ k-s\end{array}\right)$; (3) if $s=k$, then $w_{\Lambda}=v^{\Lambda}, W_{k}=\mathbb{F}_{p}^{V}$ and $E\left(W_{k}\right)=\langle\boldsymbol{\jmath}\rangle^{\perp}$

(4) $(k-s) w_{\Lambda}=\sum_{a \in \Omega \backslash \Lambda} w_{\Lambda \cup\{a\}}$ for $0 \leq s \leq k-1$; (5) $\sum_{\Lambda \in \Omega\{s\}} w_{\Lambda}=\left(\begin{array}{l}k \\ s\end{array}\right) \boldsymbol{\jmath}$ for $1 \leq s \leq k$;

(6) if $u \in W_{s}, u=\sum_{\Lambda \in \Omega\{s\}} \alpha_{\Lambda} w_{\Lambda}$, then $u(x)=\sum_{\Lambda \subset x} \alpha_{\Lambda}$, a sum of $\left(\begin{array}{l}k \\ s\end{array}\right)$ terms $\alpha_{\Lambda}$

(7) for all $1 \leq s \leq k-1, E\left(W_{s}\right) \subseteq\langle\boldsymbol{\jmath}\rangle^{\perp}$, and if $W_{s}=E\left(W_{s}\right)$ then $p \mid\left(\begin{array}{l}n-s \\ k-s\end{array}\right)$.

From Identities $1(4)$, we see that if $p \nmid(k-s)$ then $W_{s} \subset W_{s+1}$, and thus if $p \nmid 2,3, \ldots k-1$ then

$$
\langle\boldsymbol{\jmath}\rangle=W_{0} \subset W_{1} \subset W_{2} \subset \ldots W_{k-1} \subset W_{k}=\mathbb{F}_{p}^{V},
$$


which is true, for example, if $p>k$.

For the code $W_{\Pi}$ we make use of partitions of subsets of size $2 k$ of $\Omega$. For the $2 k$-set $\Lambda=\left\{a_{1,1} a_{1,2}, a_{2,1}, a_{2,2} \ldots, a_{k, 1}, a_{k, 2}\right\}$ let

$$
\left[\left[a_{1,1} a_{1,2}\right],\left[a_{2,1}, a_{2,2}\right], \ldots,\left[a_{k, 1}, a_{k, 2}\right]\right]
$$

be the partition $\pi$, then the word $w_{\pi}$, of weight $2^{k}$ is given by

$$
w_{\pi}=\sum \pm v^{\left\{a_{1, i_{1}}, a_{2, i_{2}}, \ldots, a_{k, i_{k}}\right\}}
$$

where all the subsets of $\Lambda$ of $k$-sets of the form $\left\{a_{1, i_{1}}, a_{2, i_{2}}, \ldots, a_{k, i_{k}}\right\}$ where $i_{j} \in$ $\{1,2\}$, are present, with the sign being determined by giving $\left\{a_{1,1}, a_{2,1}, \ldots, a_{k, 1}\right\}$ the sign "+", and then demanding that any other $k$-set in the support with intersection of size $k-1$ with this set will have sign "-", and then applying this in general to get the signs on all the $2^{k}$ vertices. Then

$$
\left.W_{\Pi}=\left\langle w_{\pi}\right| \pi \text { a partition of } \Lambda \subset \Omega,|\Lambda|=2 k\right\rangle .
$$

For example, if $k=3, \Lambda=\left\{a_{1}, a_{2}, b_{1}, b_{2}, c_{1}, c_{2}\right\}, \pi=\left[\left[a_{1}, a_{2}\right],\left[b_{1}, b_{2}\right],\left[c_{1}, c_{2}\right]\right]$, then with

$$
\begin{gathered}
X=\left\{\left\{a_{1}, b_{1}, c_{1}\right\},\left\{a_{1}, b_{2}, c_{2}\right\},\left\{a_{2}, b_{1}, c_{2}\right\},\left\{a_{2}, b_{2}, c_{1}\right\}\right\} \\
X^{c}=\left\{\left\{a_{2}, b_{2}, c_{2}\right\},\left\{a_{2}, b_{1}, c_{1}\right\},\left\{a_{1}, b_{2}, c_{1}\right\},\left\{a_{1}, b_{1}, c_{2}\right\}\right\}, \\
w_{\pi}=\sum_{x \in X} v^{x}-\sum_{x \in X^{c}} v^{x} .
\end{gathered}
$$

Alternatively the words can be defined inductively. For example, from $k=3$ to $k=4$, with the extra partition set $\left[d_{1}, d_{2}\right]$ by adjoining $d_{1}$ to all the elements of the sets $X$ and $X^{c}$, keeping the same signs, and then do the same with $d_{2}$, but switching the signs. Another interpretation takes the $2^{k}$ vertices in the support of $w_{\pi}$ as the vertices of the $k$-cube, $\mathcal{Q}_{k}$, i.e. the Hamming graph $H(k, 2)$, with alternate signs on the vertices.

Lemma 1 For all $n, k, p$, for all $1 \leq s \leq k-1, W_{\Pi} \subseteq W_{s}^{\perp}$.

Proof: Clear.

$3.1 W_{1}$

In $W_{1}$ we write, for $a \in \Omega, w_{a}$ instead of $w_{\{a\}}$. We have $\operatorname{wt}\left(w_{a}\right)=\left(\begin{array}{c}n-1 \\ k-1\end{array}\right)$.

Lemma 2 For all $n \geq 2 k, \operatorname{dim}\left(W_{1}\right)=n$ if $p \nmid k$ and $\operatorname{dim}\left(W_{1}\right)=n-1$ if $p \mid k$. 
Proof: Let $u=\sum_{a} \alpha_{a} w_{a}=0$. Then for $x=\left\{a_{1}, \ldots, a_{k-1}, a_{k}\right\}, u(x)=$ $\sum_{i=1}^{k} \alpha_{a_{i}}=0$. For $y=\left\{a_{1}, \ldots, a_{k-1}, b_{k}\right\}$ we have $\sum_{i=1}^{k-1} \alpha_{a_{i}}+\alpha_{b_{k}}=0$, so subtracting gives $\alpha_{a_{k}}=\alpha_{b_{k}}$ and hence all the coefficients are the same, $\alpha_{a}=\alpha$ for all $a$. So $u=\alpha \sum_{a} w_{a}=\alpha k \boldsymbol{J}=0$, and hence $\alpha k=0$. If $p \not k$ then $\alpha=0$ and the $w_{a}$ are linearly independent and $\operatorname{dim}\left(W_{1}\right)=n$; if $p \mid k$ then $\sum_{a} w_{a}=0$, and $\operatorname{dim}\left(W_{1}\right) \leq n-1$ and spanned by the $w_{i}$ for $i<n$, say. Suppose $u=\sum_{i=1}^{n} \alpha_{i} w_{i}=0$, where $\alpha_{n}=0$. If $x=\left\{a_{1}, \ldots, a_{k-1}, a_{k}\right\}$ then $u(x)=\sum_{i=1}^{k} \alpha_{a_{i}}=0$. If $a_{i} \neq n$ for $1 \leq i \leq k$, and $y=\left\{a_{1}, \ldots, a_{k-1}, n\right\}$ then we have $\alpha_{a_{k}}=0$ for all $a_{k}$ and so the $w_{i}$ for $i<n$ are linearly independent.

Lemma 3 For $p=2, k \geq 2, n \geq 2 k$, the non-zero words of $W_{1}$ have weight $n_{r}$ for $1 \leq r \leq\lfloor n / 2\rfloor$ where

$$
n_{r}=\sum_{i=0}^{\lfloor(k-1) / 2\rfloor}\left(\begin{array}{c}
r \\
2 i+1
\end{array}\right)\left(\begin{array}{c}
n-r \\
k-(2 i+1)
\end{array}\right)
$$

and also of weight $\left(\begin{array}{l}n \\ k\end{array}\right)-n_{r}$ if $k$ is odd. There are $\left(\begin{array}{l}n \\ r\end{array}\right)$ of weight $n_{r}$.

Proof: Since $\sum_{a \in \Omega} w_{a}=k \boldsymbol{\jmath}$, we have $\boldsymbol{\jmath} \in W_{1}$ for $k$ odd. Otherwise $\sum_{a \in \Omega} w_{a}=$ 0 , and in either case we need only take up to $\lfloor n / 2\rfloor$ sums of the $w_{a}$, as long as we take the differences with $\boldsymbol{\jmath}$ as well, in the odd case.

Arguing exactly as in [14, Lemma 6], taking, for $\Delta=\left\{a_{1}, \ldots, a_{r}\right\}$ and adding the corresponding vectors $w_{a_{i}}$, we have, for $w=\sum_{i=1}^{r} w_{a_{i}} \in W_{1}$,

$$
\begin{aligned}
& w=\sum_{i=1}^{r}\left(\sum_{x_{1}, \ldots, x_{k-1} \notin \Delta} v^{\left\{a_{i}, x_{1}, \ldots, x_{k-1}\right\}}+\sum_{i_{1} \neq i, x_{1}, \ldots, x_{k-2} \notin \Delta} v^{\left\{a_{i}, a_{i_{1}}, x_{1}, \ldots, x_{k-2}\right\}}+\right. \\
& \left.\sum_{i_{1}, i_{2} \neq i ; x_{1}, \ldots, x_{k-3} \notin \Delta} v^{\left\{a_{i}, a_{i_{1}}, a_{i_{2}}, x_{1}, \ldots, x_{k-3}\right\}}+\ldots+\sum_{i_{1}, \ldots, i_{k-1} \neq i} v^{\left\{a_{i}, a_{i_{1}}, \ldots, a_{i_{k-1}}\right\}}\right),
\end{aligned}
$$

and counting gives the stated formula. If $k$ is odd then the vectors $\boldsymbol{J}-w$ must also be included.

Lemma 4 For $n \geq 2 k, k \geq 3$, p prime, the minimum weight of $W_{1}^{\perp}$ is 4 . For $k=2$, the minimum weight of $W_{1}^{\perp}$ is 4 for $p>3$, and 3 for $p=2$.

Proof: For $k \geq 3$, the word

$$
v^{\{1, . ., k-2, k-1, k\}}+v^{\{1, . ., k-2, k+1, k+2\}}-v^{\{1, . ., k-2, k-1, k+1\}}-v^{\{1, . ., k-2, k, k+2\}}
$$

is clearly in $W_{1}^{\perp}$. Obviously there can be no words of weight 1 or 2 in $W_{1}^{\perp}$ and that there can be no words of weight 3 follows from a simple argument.

For $k=2$, the word of weight 4 is $v^{\{k-1, k\}}+v^{\{k+1, k+2\}}-v^{\{k-1, k+1\}}-$ $v^{\{k, k+2\}}$, but for $p=2$ the word $v^{\{1,2\}}+v^{\{1,3\}}+v^{\{2,3\}}$ is in $W_{1}^{\perp}$. 
Lemma 5 For $n \geq 5, k \geq 2, n \geq 2 k$, any $p, W_{1}=E\left(W_{1}\right)$ if and only if $p \mid k$ and $p \nmid n$.

If $k=3$ and $p \geq 5$, then $E\left(W_{1}\right) \subset W_{1}$.

Proof: Recall that $k \boldsymbol{\jmath}=\sum_{i=1}^{n} w_{i}$.

Suppose $p \mid k$. Then $w_{1}=-\sum_{i=2}^{n} w_{i}=\sum_{i=2}^{n}\left(w_{1}-w_{i}\right)-(n-1) w_{1}$. Thus $n w_{1}=\sum_{i=2}^{n}\left(w_{1}-w_{i}\right)$, so if $p \nmid n$ then $w_{1} \in E\left(W_{1}\right)$, so $W_{1}=E\left(W_{1}\right)$.

Suppose $w_{1} \in E\left(W_{1}\right)$. Then $w_{1}=\sum_{i=2}^{n} \alpha_{i}\left(w_{1}-w_{i}\right)$, for some $\alpha_{i} \in \mathbb{F}_{p}$, and thus $u=\left(\sum_{i=2}^{n} \alpha_{i}-1\right) w_{1}-\sum_{i=2}^{n} \alpha_{i} w_{i}=0$. Thus $u(x)=0$ for every $x \in V$. For $x=\left\{1, a_{2}, \ldots, a_{k-1}, a_{k}\right\}$ and $y=\left\{1, a_{2}, \ldots, a_{k-1}, b_{k}\right\}$ this gives

$$
\left(\sum_{i=2}^{n} \alpha_{i}-1\right)-\sum_{i \in\left\{a_{2}, \ldots, a_{k}\right\}} \alpha_{i}=0 \text { and }\left(\sum_{i=2}^{n} \alpha_{i}-1\right)-\sum_{i \in\left\{a_{2}, \ldots, b_{k}\right\}} \alpha_{i}=0 .
$$

Thus $\alpha_{i}=\alpha$, a constant for all $i \geq 2$, and $w_{1}=\alpha \sum_{i=2}^{n}\left(w_{1}-w_{i}\right)$ where we assume $\alpha \neq 0$.

Thus $u=((n-1) \alpha-1) w_{1}-\alpha \sum_{i=2}^{n} w_{i}=((n-1) \alpha-1) w_{1}-\alpha\left(\sum_{i=1}^{n} w_{i}-\right.$ $\left.w_{1}\right)=((n-1) \alpha-1) w_{1}-\alpha k \boldsymbol{\jmath}+\alpha w_{1}=0$, and so $(n \alpha-1) w_{1}=\alpha k \boldsymbol{\jmath}$. Both sides of this equation must be zero, so $p \mid k$ and $n=\alpha^{-1}$, so that $p \nmid n$.

The last statement follows.

$3.2 W_{2}$

In $W_{2}$ we write, for $a, b \in \Omega, w_{a, b}$ for $w_{\{a, b\}}$. Recall that $\operatorname{wt}\left(w_{a, b}\right)=\left(\begin{array}{l}n-2 \\ k-2\end{array}\right)$.

Lemma 6 For $k=3$, all $n \geq 6$, if $p>3$ then $W_{2}$ is $a\left[\left(\begin{array}{l}n \\ 3\end{array}\right),\left(\begin{array}{l}n \\ 2\end{array}\right), n-2\right]_{p}$ code and for $n \geq 8$ any word of weight $n-2$ is a scalar multiple of $w_{a, b}$ for some $a, b \in \Omega$. For $n=7$ and $p \geq 3$, the word with support

$$
\{\{1,2,3\},\{1,2,4\},\{1,3,4\},\{2,3,4\},\{5,6,7\}\}
$$

is in $W_{2}$.

Proof: For $k=3$, if $u=\sum_{a, b} \alpha_{a, b} w_{a, b}$, then $u(\{a, b, c\})=\alpha_{a, b}+\alpha_{a, c}+\alpha_{b, c}$.

If $u=0$ then $\alpha_{a, b}=-\left(\alpha_{a, c}+\alpha_{b, c}\right)=-\left(\alpha_{a, d}+\alpha_{b, d}\right)$, and thus $2 \alpha_{a, b}=$ $-\left(\alpha_{a, c}+\alpha_{b, c}+\alpha_{a, d}+\alpha_{b, d}\right)=-\left(-\alpha_{c, d}-\alpha_{c, d}\right)=2 \alpha_{c, d}$. So if $p \neq 2, \alpha_{a, b}=$ $\alpha_{c, d}=\alpha_{a, e}$ for all $a, b, c, d, e$, and $\alpha_{a, b}=\alpha$ a constant.

Thus $u=\sum_{a, b} \alpha w_{a, b}=\left(\begin{array}{l}k \\ 2\end{array}\right) \alpha \boldsymbol{\jmath}=3 \alpha \boldsymbol{\jmath}$ since $k=3$, and thus if $p \neq 3, u=0$ only if $\alpha=0$. So $\operatorname{dim}\left(W_{2}\right)=\left(\begin{array}{l}n \\ 2\end{array}\right)$ as asserted.

For the statement about the minimum weight, the proofs of the minimum weight and support of Propositions 4,5 of [15] hold for all odd $p$, as can be checked. For $n=7$ and $p \geq 3$, it can be verified that

$$
\begin{aligned}
w_{1,2}+ & w_{1,3}+w_{1,4}+w_{2,3}+w_{2,4}+w_{3,4}+w_{5,6}+w_{5,7}+w_{6,7}-\boldsymbol{\jmath} \\
& =2\left(v^{\{1,2,3\}}+v^{\{1,2,4\}}+v^{\{1,3,4\}}+v^{\{2,3,4\}}+v^{\{5,6,7\}}\right),
\end{aligned}
$$

of weight $5=n-2$. 
Note 1 For $k=3, n \geq 7, p=2,3$ are covered in $[12,11,14,15]$. For $n=6$, for $p=2$, [12, Lemma 1] holds also for $n=6$, giving the dimension as $\left(\begin{array}{c}n-1 \\ 2\end{array}\right)=10$. For $p=3$, the proof of Lemma 6 above shows that $\operatorname{dim}\left(W_{2}\right)=\left(\begin{array}{l}n \\ 2\end{array}\right)-1$.

Lemma 7 For $n \geq 2 k, k \geq 4$, and any prime $p$ :

$$
\operatorname{dim}\left(W_{2}\right)=\left\{\begin{array}{l}
\left(\begin{array}{l}
n \\
2
\end{array}\right) \text { if } p \nmid(k-1), p \nmid\left(\begin{array}{l}
k \\
2
\end{array}\right) ; \\
\left(\begin{array}{l}
n \\
2
\end{array}\right)-1 \text { if } p \nmid(k-1), p \mid\left(\begin{array}{l}
k \\
2
\end{array}\right) ; \\
\left(\begin{array}{l}
n \\
2
\end{array}\right)-n \text { if } p|(k-1), p|\left(\begin{array}{l}
k \\
2
\end{array}\right) ; \\
\left(\begin{array}{l}
n \\
2
\end{array}\right)-n+1 \text { if } p \mid(k-1), p \nmid\left(\begin{array}{l}
k \\
2
\end{array}\right), \text { i.e. } p=2, k \equiv 3(\bmod 4) .
\end{array}\right.
$$

Proof: (1): Let $p \nmid(k-1)$.

Suppose $u=\sum_{a, b} \alpha_{a, b} w_{a, b}=0$. Then for $x \in V, x=\left\{a_{1}, \ldots, a_{k}\right\}, u(x)=$ $\sum_{a, b \in x} \alpha_{a, b}=0$. For $y=\left\{a_{1}, \ldots, a_{s}\right\} \in \Omega^{\{s\}}$, any $s \leq k, a \notin y$, let $f_{y}(a)=$ $\sum_{i=1}^{s} \alpha_{a, a_{i}}$

Now take $s=k-1, x=y \cup\{a\}$. Then

$$
u(x)=0=\sum_{b, c \in y} \alpha_{b, c}+f_{y}(a),
$$

so $f_{y}(a)$, depends only on $y$ and not on $a$, so we write it now as $f_{y}$. Let $z=\left\{b_{1}, \ldots, b_{k-1}\right\}$ be such that $y \cap z=\emptyset$. Then $f_{z}=\sum_{i=1}^{k-1} \alpha_{a_{j}, b_{i}}$ for $j=$ $1, \ldots, k-1$. Summing these $k-1$ equations gives $(k-1) f_{z}=(k-1) f_{y}$ and hence $f_{z}=f_{y}$ if $p \nmid(k-1)$. Thus in this case $f_{y}=\beta$, a constant for all $(k-1)$-subsets $y$. Taking now $z=\left\{a_{1}, \ldots, a_{k-2}, b\right\}$, for $b \neq a_{k-1}$, and $a \notin y, z$, we have

$$
\beta=\sum_{i=1}^{k-2} \alpha_{a, a_{i}}+\alpha_{a, a_{k-1}}=\sum_{i=1}^{k-2} \alpha_{a, a_{i}}+\alpha_{a, b}
$$

so that $\alpha_{a, a_{k-1}}=\alpha_{a, b}$, and hence $\alpha_{i, j}=\alpha$, a constant for all $i, j$.

Thus $u=\alpha \sum_{a, b} w_{a, b}=\alpha\left(\begin{array}{c}k \\ 2\end{array}\right) \boldsymbol{\jmath}=0$, so if $p \nmid\left(\begin{array}{l}k \\ 2\end{array}\right)$ then $\alpha=0$ and $\operatorname{dim}\left(W_{2}\right)=$ $\left(\begin{array}{l}n \\ 2\end{array}\right)$. If $p \mid\left(\begin{array}{l}k \\ 2\end{array}\right)$ but $p \nmid(k-1)$ then $\operatorname{dim}\left(W_{2}\right)=\left(\begin{array}{c}n \\ 2\end{array}\right)-1$.

(2): Let $p \mid(k-1)$.

Then $\sum_{i=2}^{n} w_{1, i}=0$, so $W_{2}=\left\langle w_{a, b} \mid a, b \in \Omega \backslash\{n\}\right\rangle$, and $\operatorname{dim}\left(W_{2}\right) \leq\left(\begin{array}{c}n-1 \\ 2\end{array}\right)$. Since $\sum_{a, b} w_{a, b}=\left(\begin{array}{c}k \\ 2\end{array}\right) \boldsymbol{\jmath}$, if $p \mid\left(\begin{array}{l}k \\ 2\end{array}\right)$ then $\sum_{a, b} w_{a, b}=\sum_{a, b \neq n} w_{a, b}+\sum_{a, n} w_{a, n}=0$, so since $\sum_{a, n} w_{a, n}=0$, the $\left\{w_{a, b} \mid a, b \neq n\right\}$ are not linearly independent. We show that removing another arbitrary $w_{a, b}$, say $w_{n-2, n-1}$ would produce a linearly independent set.

Thus assuming $p \mid(k-1)$ and $p \mid\left(\begin{array}{c}k \\ 2\end{array}\right)$, let $u=\sum_{a, b} \alpha_{a, b} w_{a, b}=0$, where $\alpha_{i, n}=\alpha_{n-1, n-2}=0$ for all $i$. Then if $x=\{1, \ldots, k\}, u(x)=\sum_{1 \leq i, j \leq k} \alpha_{i, j}=0$. Similarly, for $y=\{1, \ldots, k-1, n\}$ we have $\sum_{1 \leq i, j<k-1} \alpha_{i, j}=0$, so for any $(k-1)$-set $z$ not containing $n$, we have $\sum_{a, b \in z} \alpha_{a, b}=0$. This also implies that $\sum_{i=1}^{k-1} \alpha_{i, k}=0$, i.e. in the notation above, for any $(k-1)$-set $z$ not containing $n, f_{z}(a)=0$ for all $a \notin z$. Thus if $z_{1}=\left\{a_{1}, \ldots a_{k-2}, a\right\}$ and $z_{2}=\left\{a_{1}, \ldots a_{k-2}, b\right\}$, none of the $a_{i}, a, b$ equal to $n$, then $\sum_{i=1}^{k-2} \alpha_{a_{i}, b}+\alpha_{a, b}=$ 
$0=\sum_{i=1}^{k-2} \alpha_{a_{i}, b}+\sum_{i, j} \alpha_{a_{i}, a_{j}}$, so that $\alpha_{a, b}=\sum_{i, j} \alpha_{a_{i}, a_{j}}$, and is thus a constant over $\Omega \backslash\{n\}$. Since $\alpha_{n-2, n-1}=0$ this shows that $\alpha_{a, b}=0$ for all $a, b \in \Omega \backslash\{n\}$, and thus $\operatorname{dim}\left(W_{2}\right)=\left(\begin{array}{l}n \\ 2\end{array}\right)-n$.

If $p \nmid\left(\begin{array}{c}k \\ 2\end{array}\right)$ then clearly $p=2$ and $k \equiv 3(\bmod 4)$. Let $u=\sum_{a, b} \alpha_{a, b} w_{a, b}=0$, where $\alpha_{i, n}=0$ for all $i$. The identical argument to the above yields that $\alpha_{a, b}=$ $\alpha$ is a constant. Thus $u=\alpha \sum_{a, b \neq n} w_{a, b}=0$, and since $w=\sum_{a, b \neq n} w_{a, b}$ has $w(x)=1$ if $n \notin x, w \neq 0$, so $\alpha=0$ and we have the stated result.

Lemma 8 For $n \geq 7, k \geq 3, n \geq 2 k$, any $p, W_{2}=E\left(W_{2}\right)$ if and only if

$$
p \mid\left(\begin{array}{l}
k \\
2
\end{array}\right) \text { and } p \nmid\left(\begin{array}{l}
n \\
2
\end{array}\right) \text {, or, } p \mid(k-1) \text { and } p \nmid(n-1) \text {. }
$$

For $k=3, p \geq 5, E\left(W_{2}\right) \neq W_{2}$, and if $n \geq 9$ the minimum weight of $E\left(W_{2}\right)$ is $2(n-3)$.

Proof: Suppose $W_{2}=E\left(W_{2}\right)$. Then $w_{1,2}=\sum_{\{c, d\} \neq\{1,2\}} \alpha_{c, d}\left(w_{1,2}-w_{c, d}\right)$, for some $\alpha_{c, d} \in \mathbb{F}_{p}$. Thus

$$
u=w_{1,2}\left(1-\sum_{c, d} \alpha_{c, d}\right)+\sum_{c, d} \alpha_{c, d} w_{c, d}=\sum_{a, b} \beta_{a, b} w_{a, b}=0
$$

where $\beta_{1,2}=1-\sum_{c, d} \alpha_{c, d}$, and $\beta_{c, d}=\alpha_{c, d}$ otherwise.

From the proof of Lemma 7 , if $p \nmid(k-1)$ then $\beta_{c, d}=\beta$, a constant for all $\{c, d\}$. Thus $\left(1-\left(\left(\begin{array}{l}n \\ 2\end{array}\right)-1\right) \beta\right)=\beta$, so that $\left(\begin{array}{l}n \\ 2\end{array}\right) \beta=1$. Thus $\left(\begin{array}{l}n \\ 2\end{array}\right)=\beta^{-1} \neq 0$. We have $u=\beta \sum_{a, b} w_{a, b}=\beta\left(\begin{array}{c}k \\ 2\end{array}\right) \boldsymbol{\jmath}=0$, so we must have $p\left(\begin{array}{c}k \\ 2\end{array}\right)$. Here we have, conversely, that if $p \mid\left(\begin{array}{l}k \\ 2\end{array}\right)$ then $\sum_{a, b} w_{a, b}=\left(\begin{array}{c}k \\ 2\end{array}\right) \boldsymbol{J}=0$, so $\sum_{a, b} w_{a, b}=0$ and $w_{1,2}=-\sum_{\{a, b\} \neq\{1,2\}} w_{a, b}=\sum_{\{a, b\} \neq\{1,2\}}\left(w_{1,2}-w_{a, b}\right)-\left(\left(\begin{array}{l}n \\ 2\end{array}\right)-1\right) w_{1,2}$, so $\left(\begin{array}{l}n \\ 2\end{array}\right) w_{1,2}=\sum_{\{a, b\} \neq\{1,2\}}\left(w_{1,2}-w_{a, b}\right)$ and thus if $p \nmid\left(\begin{array}{l}n \\ 2\end{array}\right)$, and $p \mid\left(\begin{array}{c}k \\ 2\end{array}\right)$, then $W_{2}=E\left(W_{2}\right)$.

If $p \mid(k-1)$ then $\sum_{i=2}^{n} w_{1, i}=0$, so $w_{1,2}=-\sum_{i=3}^{n} w_{1, i}=\sum_{i=3}^{n}\left(w_{1,2}-\right.$ $\left.w_{1, i}\right)-(n-2) w_{1,2}$. Thus $(n-1) w_{1,2}=\sum_{i=3}^{n}\left(w_{1,2}-w_{1, i}\right)$, so $w_{1,2} \in E\left(W_{2}\right)$ if $p \nmid(n-1)$.

Finally we show that if $p \mid(k-1)$ and $p \mid(n-1)$ then $W_{2} \neq E\left(W_{2}\right)$. We can assume that $W_{2}=\left\langle w_{a, b} \mid a, b<n\right\rangle$. Thus if $w_{1,2}=\sum_{\{c, d\} \neq\{1,2\}} \alpha_{c, d}\left(w_{1,2}-\right.$ $\left.w_{c, d}\right)$, for some $\alpha_{c, d} \in \mathbb{F}_{p}$, we take $\alpha_{c, d}=0$ if $c$ or $d$ is $n$. Thus

$$
u=w_{1,2}\left(1-\sum_{c, d} \alpha_{c, d}\right)+\sum_{c, d} \alpha_{c, d} w_{c, d}=\sum_{a, b} \beta_{a, b} w_{a, b}=0
$$

where $\beta_{1,2}=1-\sum_{c, d} \alpha_{c, d}$, and $\beta_{c, d}=\alpha_{c, d}$ otherwise, and $\beta_{a, n}=0$ for all $1 \leq a \leq n-1$. As in the proof of Lemma 7 , we have $f_{y}(a)=\sum_{i=1}^{k-1} \beta_{a, a_{i}}$, where $y=\left\{a_{1}, \ldots, a_{k-1}\right\}$, is independent of $a$, so taking $a=n$ gives $f_{y}=0$ for any $y$ not containing $n$. So inside $\Omega \backslash\{n\}$, taking $z=\left\{a_{1}, \ldots, a_{k-2}, b_{k-1}\right\}$, and $a \notin y, z$ we have $f_{y}(a)=f_{z}(a)=0$, so $\beta_{a, a_{k}}=\beta_{a, b_{k}}$, and it follows that $\beta_{a, b}=\beta$ for all $a, b \in \Omega \backslash\{n\}$. Thus $\beta_{1,2}=1-\sum_{c, d} \beta=\beta$ where $c, d$ ranges over 
$\Omega \backslash\{n\}$, and $\{c, d\} \neq\{1,2\}$. Thus $\left(1-\beta\left(\left(\begin{array}{l}n \\ 2\end{array}\right)-n\right)\right)=\beta$, so $1=(n-1) \beta\left(\frac{n}{2}-1\right)$, which is not possible if $p \mid(n-1)$. So here $W_{2} \neq E\left(W_{2}\right)$.

If $k=3$ and $p \geq 5$ then clearly we have $W_{2} \neq E\left(W_{2}\right)$. The statement about the minimum weight follows from [15, Proposition 6], since that proof is purely combinatorial and does not rely on the value of $p$.

Lemma 9 For all $p$, all $k \geq 2$, and $n \geq 2 k+1, \operatorname{dim}\left(W_{k-1}\right) \geq\left(\begin{array}{l}n-1 \\ k-1\end{array}\right)$. For $p=2$ we have equality.

Proof: It is easy to see that $S=\left\{w_{\Lambda}|| \Lambda \mid=k-1, n \notin \Lambda\right\}$ is a linearly independent set for any $p$, and thus $\operatorname{dim}\left(W_{k-1}\right) \geq\left(\begin{array}{l}n-1 \\ k-1\end{array}\right)$. For $p=2$, for any $\Lambda \in \Omega^{\{k-2\}}$, by Identities $1(4),(k-(k-2)) w_{\Lambda}=\sum_{a \notin \Lambda} w_{\Lambda \cup\{a\}}=0$, so $W_{k-1}=\left\langle w_{\Lambda}|| \Lambda \mid=k-1, n \notin \Lambda\right\rangle$, and thus $\operatorname{dim}\left(W_{k-1}\right) \leq\left(\begin{array}{l}n-1 \\ k-1\end{array}\right)$, so that we have equality.

\section{$4 W_{\Pi}$}

In [14, Lemma 10] for $k=3$ a list of $\left(\begin{array}{l}n \\ 3\end{array}\right)-\left(\begin{array}{l}n \\ 2\end{array}\right)$ words $w_{\pi}$ were given such that with a specified ordering of the vertices of the graph (i.e. the triples), a matrix was produced in upper-triangular form with no zero elements on the diagonal. An analogous choice can be made for $k=2$ to get a matrix in upper triangular form: see [8, Lemma 5.2.7]. Thus over any field the span of this matrix for $k=2,3$ has rank at least $\left(\begin{array}{l}n \\ k\end{array}\right)-\left(\begin{array}{c}n \\ k-1\end{array}\right)$, and thus we can assert:

Result 1 For $n \geq 5$ for $k=2$, and for $n \geq 7$ and $k=3$, any prime $p$, $\operatorname{dim}\left(W_{\Pi}\right) \geq\left(\begin{array}{l}n \\ k\end{array}\right)-\left(\begin{array}{c}n \\ k-1\end{array}\right)$

This gives:

Lemma 10 For $n \geq 7, k=3$, and any prime $p, \operatorname{dim}\left(W_{\Pi}\right)=\left(\begin{array}{l}n \\ 3\end{array}\right)-\left(\begin{array}{l}n \\ 2\end{array}\right)$, and $W_{2}=W_{\Pi}^{\perp}$. For $n \geq 5, k=2$, and any prime $p, \operatorname{dim}\left(W_{\Pi}\right)=\left(\begin{array}{c}n \\ 2\end{array}\right)-\left(\begin{array}{c}n \\ 1\end{array}\right)$; if $p>2$ then $W_{\Pi}=W_{1}^{\perp}$, and if $p=2, W_{\Pi} \subset W_{1}^{\perp}$.

Proof: For $k=3$ and $p=2,3$ this is proved in [12,11]. For $p \geq 5$, from Lemma 6 , we have $\operatorname{dim}\left(W_{2}\right)=\left(\begin{array}{c}n \\ 2\end{array}\right)$. Now $W_{2} \subseteq W_{\Pi}^{\perp}$, so $\operatorname{dim}\left(W_{\Pi}^{\perp}\right) \geq\left(\begin{array}{c}n \\ 2\end{array}\right)$. Now from Result 1 we have $\operatorname{dim}\left(W_{\Pi}^{\perp}\right) \leq\left(\begin{array}{c}n \\ 2\end{array}\right)$, and thus we have equality. A similar argument works for $k=2$ by Lemma 2 .

As in the case of $k=3$ (see $[12,11,14,15]$ ), we have the following:

Proposition 1 For $n \geq 2 k+1$, any prime $p$, and any $k \geq 2$, if $C$ is a code such that $W_{\Pi} \subseteq C \subseteq \mathbb{F}_{p}^{V}$, where $|V|=\left(\begin{array}{l}n \\ k\end{array}\right)$, then $C^{\perp}$ has minimum weight at least $n-k+1$.

Proof: Let $\mathcal{B}=\left\{\operatorname{Supp}\left(w_{\pi}\right) \mid w_{\pi} \in W_{\Pi}\right\}$. Then $\mathcal{D}=(V, \mathcal{B})$ is a $1-\left(\left(\begin{array}{l}n \\ k\end{array}\right), 2^{k}, r\right)$ design where $r$ is the number of blocks of $\mathcal{B}$ through a point. We have $r=$ $\left(\begin{array}{c}n-k \\ k\end{array}\right) k$ !, since for $x \in V$ there are $\left(\begin{array}{c}n-k \\ k\end{array}\right)$ ways of choosing a $k$-set to complete 
to a $2 k$-set, and there are then $k$ ! ways to choose the partition such that $x$ is in the support.

The number of blocks of $\mathcal{B}$ that contain two distinct points $x, y$ can have $k$ values, depending on the size of $|x \cap y|$. For a fixed point $x$, for $0 \leq i \leq k-1$, if $y \neq x$, and $|x \cap y|=i$, let $n_{i}$ denote the number of blocks of $\mathcal{B}$ that contain both $x$ and $y$. Then counting yields that $n_{i}=\left(\begin{array}{c}n-2 k+i \\ i\end{array}\right) i !(k-i)$ !. For a fixed $x$ we say a point $y$ is of type- $i$ if $|x \cap y|=i$.

Now let $S=\operatorname{Supp}(w)$ where $w \in C^{\perp}$, and $|S|=s$. Let $x \in S$. Let $z_{i}$, for $i=0$ to $2^{k}$ be the number of blocks in $\mathcal{B}$ that contain $x$ and meet $S$ in $i$ points. Then $z_{0}=z_{1}=0$, and $\sum_{i=2}^{2^{k}} z_{i}=r$. For $0 \leq i \leq k-1$, suppose there are $\ell_{i}$ points in $S \backslash\{x\}$ of type- $i$. Then counting gives

$$
\sum_{i=2}^{2^{k}}(i-1) z_{i}=\sum_{i=0}^{k-1} \ell_{i} n_{i}
$$

and $s-1=\sum_{i=0}^{k-1} \ell_{i}$.

For $n \geq 2 k+1$, it is easy to prove directly that $n_{k-1} \geq n_{i}$ for $0 \leq i \leq k-1$. Thus we have

$$
r \leq \sum_{i=2}^{2^{k}}(i-1) z_{i}=\sum_{i=0}^{k-1} \ell_{i} n_{i} \leq \sum_{i=0}^{k-1} \ell_{i} n_{k-1}=(s-1) n_{k-1} .
$$

Since $r=(n-k) n_{k-1}$, this gives $n-k \leq s-1$, i.e. $s \geq n-k+1$.

Corollary 1 For $n \geq 2 k+1$, any $k \geq 2$, p prime, for $1 \leq s \leq k-1, W_{s}$ has minimum weight at least $n-k+1$ and $W_{k-1}$ has minimum weight $n-k+1$. For $n>2 k+1$, the minimum words of $W_{k-1}$ are the scalar multiples of the $w_{\Lambda}$ for $\Lambda \subset \Omega$ with $|\Lambda|=k-1$.

Proof: Since $W_{\Pi} \subseteq W_{s}^{\perp}$ for $1 \leq s \leq k-1$, we have that $W_{s}$ has minimum weight at least $n-k+1$ by Proposition 1 . For $s=k-1=|\Lambda|, \operatorname{wt}\left(w_{\Lambda}\right)=$ $n-k+1$, so the minimum weight of $W_{k-1}$ is $n-k+1$.

Let $w \in W_{k-1}$ have weight $n-k+1$ and support $S$. With the same notation as in the proposition, and writing $\ell_{k-1}=n-k-\sum_{i=0}^{k-2} \ell_{i}$, we have

$$
r=(n-k) n_{k-1} \leq \sum_{i=0}^{k-2} n_{i} \ell_{i}+n_{k-1}\left(n-k-\sum_{i=0}^{k-2} \ell_{i}\right),
$$

so that

$$
r \leq \sum_{i=0}^{k-2}\left(n_{i}-n_{k-1}\right) \ell_{i}+r .
$$

Now $n_{k-1} \geq n_{i}$ for $0 \leq i \leq k-2$, with possible equality only if $n=2 k+1$. Thus for $n>2 k+1$ we have $\ell_{i}=0$ for $0 \leq i \leq k-2$. Thus $x \in S$ has $k-1$ elements in common with any other $y$ in $S$, and this is true for all pairs of points. 
Suppose $x=\left\{a_{1}, \ldots, a_{k-1}, a_{k}\right\} \in S$. Let $y=\left\{a_{1}, \ldots, a_{k-1}, a_{k+1}\right\} \in S$. If $\operatorname{Supp}(w) \neq \operatorname{Supp}\left(w_{\left\{a_{1}, \ldots, a_{k-1}\right\}}\right)$ and if $z \in S$ contains a further element $a_{k+2}$ of $\Omega$, but is not $\left\{a_{1}, \ldots, a_{k-1}, a_{k+2}\right\}$, then $z$ contains $a_{k}, a_{k+1}, a_{k+2}$ and hence cannot satisfy that it intersects $x, y$ in $k-1$ elements. Thus $z$ contains only elements from $\left\{a_{1}, \ldots, a_{k+1}\right\}$, and we can obtain at most $k+1$ such points. Since $n-k+1 \geq 2 k+1-k+1=k+2$, this is not the support of $S$. Thus it follows that $\operatorname{Supp}(w)=\operatorname{Supp}\left(w_{\left\{a_{1}, \ldots, a_{k-1}\right\}}\right)$.

Let $\Lambda=\left\{a_{1}, \ldots, a_{k-1}\right\}$, and suppose $w=\sum_{a \in \Omega \backslash \Lambda} \alpha_{a} v^{\Lambda \cup\{a\}}$, where $\alpha_{a} \neq$ 0 . By scaling we can take $\alpha_{a}=1$ for some $a$. Then $w-w_{\Lambda}=\sum_{b \in \Omega \backslash \Lambda}\left(\alpha_{b}-\right.$ 1) $v^{\Lambda \cup\{b\}}$ will have non-zero weight less than $n-k+1$ if not all the $\alpha_{b}$ are 1 . Thus $w$ is a scalar multiple of $w_{\Lambda}$.

\section{Hulls}

$5.1 \operatorname{Hull}\left(W_{1}\right)$

Proposition 2 For $n \geq 7, k \geq 2$, p prime, $\operatorname{Hull}\left(W_{1}\right)$ is one of $W_{1}, E\left(W_{1}\right),\langle\boldsymbol{\jmath}\rangle$ or $\{0\}$. Specifically, writing $n_{1}=\left(\begin{array}{l}n-1 \\ k-1\end{array}\right), n_{2}=\left(\begin{array}{l}n-2 \\ k-2\end{array}\right), H=\operatorname{Hull}\left(W_{1}\right)$,

1. if $n_{1} \equiv n_{2}(\bmod p) \equiv r(\bmod p)$, then if $r=0, H=W_{1}$, and if $r \neq 0$, $H=E\left(W_{1}\right)$;

2. if $n_{1} \not \equiv n_{2}(\bmod p)$, then

(a) if $n_{2} \equiv 0(\bmod p)$ then $H=\{0\}$;

(b) if $n_{1} \equiv 0(\bmod p)$ then $H=\{0\}$ if $p \mid k$, and $H=\langle\boldsymbol{\jmath}\rangle$ if $p \nmid k$;

(c) if neither $n_{1} \equiv 0(\bmod p)$ nor $n_{2} \equiv 0(\bmod p)$, then $H=\{0\}$.

If $p \geq n$ then $H=\{0\}$.

Proof: For any $a, b \in \Omega,\left(w_{a}, w_{a}\right)=\left(\begin{array}{l}n-1 \\ k-1\end{array}\right)=n_{1}$, and $\left(w_{a}, w_{b}\right)=\left(\begin{array}{l}n-2 \\ k-2\end{array}\right)=n_{2}$. Also, $\left(w_{a}, \boldsymbol{\jmath}\right)=n_{1}, \sum_{a} w_{a}=k \boldsymbol{\jmath}$, so $\boldsymbol{\jmath} \in W_{1}^{\perp}$ if $n_{1} \equiv 0(\bmod p)$, and $\boldsymbol{\jmath} \in H$ if also $p \nmid k$.

(1) Suppose that $n_{1} \equiv n_{2}(\bmod p) \equiv r(\bmod p)$. So $\left(w_{a}, w_{b}\right) \equiv r(\bmod p)$ for all $a, b \in \Omega$, so $w_{a}-w_{b} \in H$, and thus $E\left(W_{1}\right) \subseteq H$. If $r \equiv 0(\bmod p)$ then $W_{1}=H$, and if $r \not \equiv 0(\bmod p), E\left(W_{1}\right)=H$

(2) Suppose that $n_{1} \not \equiv n_{2}(\bmod p)$. Let $w=\sum_{a \in \Omega} \alpha_{a} w_{a} \in H$. Then for any $b \in \Omega,\left(w, w_{b}\right)=\alpha_{b} n_{1}+n_{2} \sum_{a \neq b} \alpha_{a}=\alpha_{b}\left(n_{1}-n_{2}\right)+n_{2} \sum_{a \in \Omega} \alpha_{a}=0$. Since $n_{1}-n_{2} \not \equiv 0(\bmod p)$, this gives $\alpha_{b}=\frac{n_{2}}{n_{2}-n_{1}} \sum_{a} \alpha_{a}$, i.e. $\alpha_{b}=\alpha$, a constant for all $b$, and $w=\alpha k \boldsymbol{\jmath}$.

(a) If $n_{2} \equiv 0(\bmod p)$ then $\alpha=0$, so $H=\{0\}$.

(b) If $n_{1} \equiv 0(\bmod p)$ then $H=\{0\}$ if $p \mid k$, and $H=\langle\boldsymbol{\jmath}\rangle$ if $p \nmid k$.

(c) If neither $n_{1} \equiv 0(\bmod p)$ nor $n_{2} \equiv 0(\bmod p)$, then $\left(w, w_{a}\right)=\alpha k\left(w_{a}, \boldsymbol{\jmath}\right)=$ $\alpha k n_{1}=0$, so $\alpha k=0$ and $w=0$.

Finally, if $p \geq n$, then (c) holds. 
Corollary 2 If $n \geq 7, k=3, p \geq 5$, then, with $H=\operatorname{Hull}\left(W_{1}\right)$, if $n \equiv 1(\bmod p)$ then $H=\langle\boldsymbol{\jmath}\rangle$; if $n \equiv 2(\bmod p)$ then $H=W_{1}$; if $n \neq \equiv 1,2(\bmod p)$ then $H=\{0\}$.

\section{$5.2 \operatorname{Hull}\left(W_{2}\right)$}

For $W_{2}$ we have, for distinct $a, b, c, d \in \Omega, k \geq 3$ :

$$
\begin{gathered}
\left(w_{a, b}, w_{a, b}\right)=\left(\begin{array}{l}
n-2 \\
k-2
\end{array}\right)=n_{2} ;\left(w_{a, b}, w_{a, c}\right)=\left(\begin{array}{l}
n-3 \\
k-3
\end{array}\right)=n_{3} \\
\left(w_{a, b}, w_{c, d}\right)=\left(\begin{array}{l}
n-4 \\
k-4
\end{array}\right)=n_{4},\left(w_{a, b}, w_{a}\right)=\left(\begin{array}{l}
n-2 \\
k-2
\end{array}\right)=n_{2} ; \\
\left(w_{a, b}, w_{c}\right)=\left(\begin{array}{l}
n-3 \\
k-3
\end{array}\right)=n_{3} ; \sum_{a, b \in \Omega} w_{a, b}=\left(\begin{array}{l}
k \\
2
\end{array}\right) \boldsymbol{\jmath} .
\end{gathered}
$$

Let $w=\sum_{a, b} \alpha_{a, b} w_{a, b} \in \operatorname{Hull}\left(W_{2}\right), \sigma=\sum_{a, b} \alpha_{a, b}$, and $\beta_{a}=\sum_{b \neq a} \alpha_{a, b}$. Then $(\boldsymbol{\jmath}, w)=n_{2} \sigma$, and

$$
\begin{gathered}
\left(w, w_{a, b}\right)=n_{2} \alpha_{a, b}+n_{3}\left(\sum_{c \neq a, b} \alpha_{a, c}+\sum_{c \neq a, b} \alpha_{b, c}\right)+n_{4} \sum_{c, d \in \Omega \backslash\{a, b\}} \alpha_{c, d}=0, \\
\left(w, w_{a}\right)=n_{2} \sum_{b \neq a} \alpha_{a, b}+n_{3} \sum_{b, c \neq a} \alpha_{b, c} .
\end{gathered}
$$

If $p \nmid(k-1)$ then $W_{1} \subseteq W_{2}$, and we get, for $w \in \operatorname{Hull}\left(W_{2}\right)$ :

1. $\left(w, w_{a, b}\right)=\left(n_{2}-2 n_{3}+n_{4}\right) \alpha_{a, b}+\left(n_{3}-n_{4}\right)\left(\beta_{a}+\beta_{b}\right)+n_{4} \sigma=0$,

2. $\left(w, w_{a}\right)=\left(n_{2}-n_{3}\right) \beta_{a}+n_{3} \sigma=0$.

Furthermore, $w=\sum_{x, y} \alpha_{x, y}\left(w_{x, y}-w_{a, b}\right)+\sigma w_{a, b}$, so if $\sigma=0$ then $w \in E\left(W_{2}\right)$.

For $k=3$ we can take $p \geq 5$, since $k=2,3$ was studied in $[12,11,14,15]$.

With notation as given above we have $W_{1} \subseteq W_{2}, n_{2}=n-2, n_{3}=1, n_{4}=0$.

Lemma 11 For $k=3, p \geq 5, H=\operatorname{Hull}\left(W_{2}\right)$, if $n \not \equiv 2,3,4(\bmod p)$ then $H=\{0\}$; if $n \equiv 2(\bmod p)$ then $H=\langle\boldsymbol{J}\rangle$; if $n \equiv 3(\bmod p)$ then $E\left(W_{1}\right) \subseteq H$; if $n \equiv 4(\bmod p)$ then for any 4 -set $\{a, b, c, d\}$ of $\Omega, w_{a, b}+w_{c, d}-w_{a, c}-w_{b, d} \in$ $H$, and has weight $4(n-4)$. Furthermore, if $p \geq n-1$ then $H=\{0\}$.

Proof: For $k=3$ the two equations above for $w=\sum_{a, b} \alpha_{a, b} w_{a, b} \in H$ become

$$
(n-4) \alpha_{a, b}+\beta_{a}+\beta_{b}=0,(n-3) \beta_{a}+\sigma=0 .
$$

If $n \not \equiv 3(\bmod p)$, then $\beta_{a}=\alpha$, a constant, and $(n-4) \alpha_{a, b}=-2 \alpha$. If $n \not \equiv 4(\bmod p)$ then $\alpha_{a, b}=\beta$, a constant, so $w=\beta\left(\begin{array}{l}k \\ 2\end{array}\right) \boldsymbol{\jmath}=3 \beta \boldsymbol{\jmath}$. Now $\left(\boldsymbol{\jmath}, w_{a, b}\right)=$ $n-2$ so $\boldsymbol{\jmath} \in W_{2}^{\perp}$ only if $n \equiv 2(\bmod p)$. Thus if $n \not \equiv 2(\bmod p)$ then $H=\{0\}$, proving the first assertion. 
If $n \equiv 2(\bmod p)$ then clearly $n \not \equiv 3,4(\bmod p)($ since $p \geq 5)$, so from the above we have $H=\langle\boldsymbol{\jmath}\rangle$.

If $n \equiv 3(\bmod p)$ then $\left(w_{a, b}, w_{a}\right)=\left(w_{a, b}, w_{c}\right) \equiv 1(\bmod p)$, so $E\left(W_{1}\right) \subseteq H$, since we already have $W_{1} \subset W_{2}$.

If $n \equiv 4(\bmod p)$ then it can be verified directly that for any 4 -set $\{a, b, c, d\}$ of $\Omega, w_{a, b}+w_{c, d}-w_{a, c}-w_{b, d} \in H$.

Finally, if $p \geq n-1$ then $p \nmid n_{2}, n_{3}, n_{4}$ and so we get $\beta_{a}=\frac{-n_{3} \sigma}{\left(n_{2}-n_{3}\right)}=\alpha$, a constant, so $\alpha_{a, b}=\beta$, a constant, and $w=\beta\left(\begin{array}{l}k \\ 2\end{array}\right) \boldsymbol{\jmath}$. If $\beta \neq 0$ then $\boldsymbol{\jmath} \in H$. But $\left(w_{a, b}, \boldsymbol{\jmath}\right)=n_{2} \neq 0$, so $\beta=0$ and $w=0$.

\section{Codes from $\Gamma(n, k, r)$ and $R \Gamma(n, k, r)$ and the $W_{i}$}

Let $A_{r}^{k}, R A_{r}^{k}=A_{r}^{k}+I$ be adjacency matrices for $\Gamma(n, k, r)$ and $R \Gamma(n, k, r)$ respectively, where $0 \leq r \leq k-1,2 \leq k \leq n / 2$. For any fixed prime $p$, let $C_{r}, R C_{r}$ denote the row span over $\mathbb{F}_{p}$ of $A_{r}^{k}, R A_{r}^{k}$, respectively. Let $V=\Omega^{\{k\}}$.

Rows of $A_{r}^{k}, R A_{r}^{k}$ for $r=0,1, \ldots, k-1$ are denoted by $r_{x}^{r}, s_{x}^{r}$ respectively, for $x \in V$, where we assume a fixed value of $k \geq 2$. Thus $s_{x}^{r}=r_{x}^{r}+v^{x}$. Also, we write

$$
N_{r}(x)=\{y \in V \mid y \stackrel{r}{\sim} x\}=\left\{y \in V \mid r_{x}^{r}(y)=1\right\} .
$$

If $C$ is any of these codes we will use the notation $E(C)$ to denote the span of the differences of the rows of the adjacency matrix $A_{i}, A_{i}+I$ over the relevant field. Thus, for example, $E\left(C_{i}\right)=\left\langle r_{x}^{i}-r_{y}^{i} \mid x, y \in V\right\rangle$, where $V=\Omega^{\{k\}}$ and $r_{x}^{i}$ are the rows of $A_{i}$

We can express the $s_{x}^{r}, r_{x}^{r}$ in terms of the words $w_{\Lambda}$. For clarity we write $w_{\Lambda}^{s}=w_{\Lambda}$ where $|\Lambda|=s$. Then $w_{\emptyset}^{0}=\boldsymbol{\jmath}$ and $w_{\Lambda}^{k}=v^{\Lambda}$. Then counting gives the following relations, for $0 \leq r \leq k-1$, and any $x \in V$ :

$$
r_{x}^{r}=\sum_{j=0}^{k-r}(-1)^{j}\left(\begin{array}{c}
j+r \\
r
\end{array}\right) \sum_{\substack{y \in V \\
|x \cap y|=j+r}} w_{x \cap y}^{j+r} ; \quad s_{x}^{r}=r_{x}^{r}+v^{x} .
$$

For $\Gamma(n, k, r)$, the eigenvalues are found from the Eberlein polynomials in $[18$, Theorem 5.1] (see also $[7,1,4,6]$ and $[4$, Theorem 4.6]) for $j=0$ to $k$ :

$$
\varepsilon_{j}=\sum_{i=\operatorname{Max}\{0, j-r\}}^{\operatorname{Min}\{j, k-r\}}(-1)^{i}\left(\begin{array}{l}
j \\
i
\end{array}\right)\left(\begin{array}{c}
k-j \\
k-r-i
\end{array}\right)\left(\begin{array}{l}
n-k-j \\
k-r-i
\end{array}\right)
$$

with multiplicity $m_{j}$ as given above.

Note that in all cases $\varepsilon_{0}=\left(\begin{array}{c}k \\ k-r\end{array}\right)\left(\begin{array}{c}n-k \\ k-r\end{array}\right)$, the valency of $\Gamma(n, k, r)$, and $\varepsilon_{k}=$ $(-1)^{k-r}\left(\begin{array}{c}k \\ k-r\end{array}\right)$.

For $r=1$, i.e. $\Gamma(n, k, 1)$, this can be written more simply, for $j=0$ to $k$ :

$$
\varepsilon_{j}=(-1)^{j-1} j\left(\begin{array}{c}
n-k-j \\
k-j
\end{array}\right)+(-1)^{j}(k-j)\left(\begin{array}{c}
n-k-j \\
k-j-1
\end{array}\right) .
$$


6.1 Kneser and Johnson graphs

The Kneser and Johnson graphs have had special attention in the literature. The graph $\Gamma(n, k, 0)$ is the Kneser graph $K G_{n, k}$. Its eigenvalues are, for $j=0$ to $k$ :

$$
\lambda_{j}=(-1)^{j}\left(\begin{array}{c}
n-k-j \\
k-j
\end{array}\right)
$$

with multiplicity $m_{j}=\left(\begin{array}{c}n \\ j\end{array}\right)-\left(\begin{array}{c}n \\ j-1\end{array}\right)$ for $j>0$ and 1 for $j=0$.

The graph $\Gamma(n, k, k-1)$ is the Johnson graph $J(n, k)$. Its eigenvalues are (see [21] and the above formulal), for $j=0$ to $k$ :

$$
\theta_{j}=k(n-k)-j(n+1-j)
$$

with multiplicity $m_{j}=\left(\begin{array}{c}n \\ j\end{array}\right)-\left(\begin{array}{c}n \\ j-1\end{array}\right)$ for $j>0$ and 1 for $j=0$.

We can use these eigenvalues to get information regarding the possible dimension of the codes $C_{0}$ and $C_{k-1}$. Since if $\lambda$ is an eigenvalue for a matrix $M$ then $\lambda+1$ is an eigenvalue for $M+I$, this will also give information about $R C_{0}$ and $R C_{k-1}$. If $M$ is a $v \times v$ integral matrix with integral eigenvalues, then modulo $p$ these will still be eigenvalues, but not necessarily all distinct. If none or at most one reduce to 0 modulo $p$ then the $p$-rank of $M$ will be $v$ or $v-m_{j}$, respectively, where $m_{j}$ is the multiplicity of the eigenvalue that is zero. In any case, the dimension of the zero eigenspace over $\mathbb{F}_{p}$ of the matrix $A$ or $A+I$ is at most the sum $m$ of the multiplicities of the eigenvalues that reduce to 0 modulo $p$, and thus the $p$-rank of $A$ or $A+I$ is at least $\left(\begin{array}{l}n \\ k\end{array}\right)-m .^{1}$

Note 2 Since $\lambda_{k}+1=(-1)^{k}+1=0$ if $k$ is odd, we see that $R C_{0} \neq \mathbb{F}_{p}^{|V|}$ for $k$ odd. In fact, notice that from Equation (6), for $x \in V=\Omega^{\{k\}}$ :

$$
s_{x}^{0}=v^{x}+\sum_{j=0}^{k}(-1)^{j} \sum_{\substack{y \in V \\|x \cap y|=j}} w_{x \cap y}^{j}=v^{x}+\boldsymbol{\jmath}+\sum_{j=1}^{k-1}(-1)^{j} \sum_{\substack{y \in V \\|x \cap y|=j}} w_{x \cap y}^{j}+(-1)^{k} v^{x},
$$

so if $k$ is odd then $s_{x}^{0}=\boldsymbol{\jmath}+\sum_{i=1}^{k-1} u_{i}$, where $u_{i} \in W_{i}$. Thus if $\boldsymbol{\jmath}, u_{i} \in W_{k-1}$, for $1 \leq i \leq k-1$, we have $R C_{0} \subseteq W_{k-1}$. So if $p \nmid 2,3, \ldots, k$, this will hold.

From [9] (Propositions 3.1, 3.2, 3.3, 3.4) we can deduce the following connection with the binary codes from the Johnson graphs, $J(n, k)$, the uniform subset graph $\Gamma(n, k, k-1)$, and from [8, Theorem 6.2.7] or [10, Theorem 3.7], for the binary code from the odd graph $\mathcal{O}(k)=\Gamma(2 k+1, k, 0)$ :

Result 2 Let $k \geq 3, n \geq 2 k+1, p=2$.

1. If $C=C_{2}(J(n, k))$, then

- for $n, k$ odd, $C=W_{k-1}^{\perp}$; for $n$ odd, $k$ even, $C=W_{k-1}$;

- for $n, k$ even, $C \subset W_{k-1}^{\perp}$; for $n$ even, $k$ odd, $C=\mathbb{F}_{2}^{V}$. 2. If $C=C_{2}(\mathcal{O}(k))$, then $C=W_{k-1}^{\perp}$.

1 The authors thank T.P. McDonough for this observation. 
From this result and the eigenvalues for $J(n, k)$ we can deduce the following for the binary codes for the Johnson graph and its associated reflexive graph:

Corollary 3 For $k \geq 3, n \geq 2 k+1, p=2$, let $C=C_{2}(J(n, k)), R C=$ $C_{2}(R J(n, k))$. Then

1. for $n, k$ odd, $R C=W_{k-1}=C^{\perp}$, $\operatorname{Hull}(C)=\{0\}$;

2. for $n$ odd, $k$ even, $R C=W_{k-1}^{\perp}=C^{\perp}$, $\operatorname{Hull}(C)=\{0\}$;

3. for $n, k$ even, $C \subseteq \operatorname{Hull}\left(W_{k-1}\right), R C=\mathbb{F}_{2}^{V}$;

4. for $n$ even, $k$ odd, $C=\mathbb{F}_{2}^{V}, R C \subseteq \operatorname{Hull}\left(W_{k-1}\right)$.

Proof: (1): By evaluating the inner products $\left(r_{x}^{k-1}, s_{y}^{k-1}\right)$ we see it has the value $k(n-k)$ if $x=y$; 0 if $0 \leq|x \cap y| \leq k-3 ; 4$ if $|x \cap y|=k-2$; and $n-1$ if $|x \cap y|=k-1$. Thus $R C \subseteq C^{\perp}$ if $n$ is odd, i.e. $R C \subseteq W_{k-1}$. Now from the eigenvalues for $J(n, k)$, we see that for $n$ odd the null space of $R C$ has dimension at most the sum of the multiplicities of the zero eigenvalues (for $A+I$ ), i.e. the sum of the $m_{i}$ for $i$ odd. This sum can be seen to be $\sum_{i=0}^{l}(-1)^{i+1}\left(\begin{array}{c}n \\ i\end{array}\right)$ where $l=k-1$ if $k$ is even, $l=k$ if $k$ is odd. Now for $l$ odd it is easy to show that

$$
\sum_{i=0}^{l}(-1)^{i+1}\left(\begin{array}{l}
n \\
i
\end{array}\right)=\left(\begin{array}{c}
n-1 \\
l
\end{array}\right),
$$

so for $k$ odd the sum is $\left(\begin{array}{c}n-1 \\ k\end{array}\right)$, and for $k$ even the sum is $\left(\begin{array}{c}n-1 \\ k-1\end{array}\right)$. Thus for $n, k$ odd, $\operatorname{dim}(R C) \geq\left(\begin{array}{l}n \\ k\end{array}\right)-\left(\begin{array}{c}n-1 \\ k\end{array}\right)=\left(\begin{array}{l}n-1 \\ k-1\end{array}\right)=\operatorname{dim}\left(W_{k-1}\right)$ by Lemma 9 . Thus $R C=W_{k-1}$.

(2): Since $n$ is still odd, we have $R C \subseteq C^{\perp}=W_{k-1}^{\perp}$. The null space again has dimension at most the sum of the $m_{i}$ for $i$ odd, but now terminating in $k-1$. So $\operatorname{dim}(R C) \geq\left(\begin{array}{l}n \\ k\end{array}\right)-\left(\begin{array}{l}n-1 \\ k-1\end{array}\right)=\left(\begin{array}{c}n-1 \\ k\end{array}\right)=\operatorname{dim}\left(W_{k-1}^{\perp}\right)$. Thus $R C=W_{k-1}^{\perp}$.

(3): For $n, k$ even, by Result $2, C \subseteq W_{k-1}^{\perp}$. By Equation (6),

$$
\begin{aligned}
& r_{x}^{k-1}=\sum_{j=0}^{1}(-1)^{j}\left(\begin{array}{c}
j+k-1 \\
k-1
\end{array}\right) \sum_{\substack{y \in V \\
|x \cap y|=j+k-1}} w_{x \cap y}^{j+k-1} \\
& =\sum_{\substack{y \in V \\
|x \cap y|=k-1}} w_{x \cap y}^{k-1}-\left(\begin{array}{c}
k \\
k-1
\end{array}\right) v^{x}=\sum_{\substack{y \in V \\
|x \cap y|=k-1}} w_{x \cap y}^{k-1}
\end{aligned}
$$

since the last term is 0 . Thus $C \subseteq \operatorname{Hull}\left(W_{k-1}\right)$. Since all the eigenvalues for $A+I$ are non-zero modulo $2, R C=\mathbb{F}_{2}^{V}$.

(4): For $n$ even, $k$ odd, the equation for $r_{x}^{k-1}$ in terms of elements from $W_{k-1}$ becomes $s_{x}^{k-1}=r_{x}^{k-1}+v^{x}=\sum_{\substack{y \in V \\|x \cap y|=k-1}} w_{x \cap y}^{k-1}$, so $R C \subseteq W_{k-1}$. Checking then that $\left(s_{x}^{k-1}, w_{z}\right) \equiv 0(\bmod 2)$ for all $x \in V, z \in \Omega^{\{k-2\}}$, we have $R C \subseteq$ $\operatorname{Hull}\left(W_{k-1}\right)$. The rest follows. 
Note 3 (1) Computations indicate that the inequalities in (3) and (4) are equalities.

(2) From Identities 1(4), we have that if $k-s$ is odd and $p=2$, then $W_{s} \subseteq$ $W_{s+1}$, for $0 \leq s \leq k-1$.

\section{Codes from $\Gamma(n, 3, i)$ and $R \Gamma(n, 3, i)$ for $p \geq 5$}

We examine the codes from the row span of adjacency matrices for $\Gamma=$ $\Gamma(n, 3, i)$ and its reflexive counterpart $R \Gamma=R \Gamma(n, 3, i)$, for $i=0,1,2$, and $p \geq 5, n \geq 7$. For $p=2,3$ these codes are studied in $[12,11,14,15]$. Recall that $\Omega=\{1, \ldots, n\}$. This section will be devoted to developing the various properties of the codes, and their links to $W_{1}, W_{2}, W_{\pi}$; the findings will be summarized in a more comprehensive manner in Section 8.

Let $V=\Omega^{\{3\}}$. Throughout this section, $n \geq 7, k=3$ and $p \geq 5$. By Lemmas 5,8 , respectively, $W_{1} \neq E\left(W_{1}\right)$, and $W_{2} \neq E\left(W_{2}\right)$.

We first obtain some general relationships amongst the words of the codes from the graphs and the words from the codes $W_{1}, W_{2}, W_{\Pi}$.

\subsection{General identities}

With notation as before, for any $x \in V$,

$$
\boldsymbol{J}=v^{x}+r_{x}^{0}+r_{x}^{1}+r_{x}^{2}=-2 v^{x}+s_{x}^{0}+s_{x}^{1}+s_{x}^{2} .
$$

The valency $\nu_{i}$ for $\Gamma(n, 3, i)$ for $i=0,1,2$ is given by:

$$
\nu_{0}=\left(\begin{array}{c}
n-3 \\
3
\end{array}\right) ; \quad \nu_{1}=3\left(\begin{array}{c}
n-3 \\
2
\end{array}\right) ; \quad \nu_{2}=3(n-3),
$$

and $\nu_{i}^{*}=\nu_{i}+1$ is the valency of $R \Gamma$.

Note 4 1. From Identities 1 (4),(5), $\boldsymbol{\jmath} \in W_{1} \subseteq W_{2}$.

2. Since $\sum_{x} r_{x}^{i}=\nu_{i} \boldsymbol{\jmath}$ and $\sum_{x} s_{x}^{i}=\nu_{i}^{*} \boldsymbol{\jmath}, \boldsymbol{\jmath} \in C_{i}$ if $\nu_{i} \not \equiv 0(\bmod p)$ and $\boldsymbol{\jmath} \in R C_{i}$ if $\nu_{i}^{*} \not \equiv 0(\bmod p)$. Furthermore, $C_{i} \subseteq\langle\boldsymbol{\jmath}\rangle^{\perp}$ if and only if $\nu_{i} \equiv 0(\bmod p)$, and $R C_{i} \subseteq\langle\boldsymbol{J}\rangle^{\perp}$ if and only if $\nu_{i}^{*} \equiv 0(\bmod p)$.

Definition 1 For $x \in \Omega^{\{3\}}, i=0,1,2$, write

$$
w_{x}^{i}=\sum_{y \in N_{i}(x) \cup\{x\}} s_{y}^{i} \text { and } u_{x}^{i}=\sum_{y \in N_{i}(x)} r_{y}^{i} .
$$

Then $w_{x}^{i}=v^{x}+2 r_{x}^{i}+u_{x}^{i}$, and, from counting arguments as detailed in [14,15, $12,11]:$

Identities $2(1) w_{x}^{0}=\left(1+\left(\begin{array}{c}n-3 \\ 3\end{array}\right)\right) v^{x}+\left(\left(\begin{array}{c}n-6 \\ 3\end{array}\right)+2\right) r_{x}^{0}+\left(\begin{array}{c}n-5 \\ 3\end{array}\right) r_{x}^{1}+\left(\begin{array}{c}n-4 \\ 3\end{array}\right) r_{x}^{2}$;

(2) $u_{x}^{0}=\left(\begin{array}{c}n-3 \\ 3\end{array}\right) v^{x}+\left(\begin{array}{c}n-6 \\ 3\end{array}\right) r_{x}^{0}+\left(\begin{array}{c}n-5 \\ 3\end{array}\right) r_{x}^{1}+\left(\begin{array}{c}n-4 \\ 3\end{array}\right) r_{x}^{2}$;

(3) $w_{x}^{1}=(60-10 n) v^{x}+9(n-6) s_{x}^{0}+\frac{1}{2}\left(n^{2}-3 n-6\right) s_{x}^{1}+(n-4)^{2} s_{x}^{2}$; 
(4) $u_{x}^{1}=3\left(\begin{array}{c}n-3 \\ 2\end{array}\right) v^{x}+9(n-6) r_{x}^{0}+\frac{(n-5)(n+2)}{2} r_{x}^{1}+(n-4)^{2} r_{x}^{2}$;

(5) $w_{x}^{2}=2(n-6) v^{x}+4 s_{x}^{1}+n s_{x}^{2}$;

(6) $u_{x}^{2}=3(n-3) v^{x}+4 r_{x}^{1}+(n-2) r_{x}^{2}$.

From Identities $1(4),(5)$, we have $\sum_{a \in \Omega} w_{a}=\sum_{a, b \in \Omega} w_{a, b}=3 \boldsymbol{\jmath}$, so, since $p \geq 5, \boldsymbol{\jmath} \in W_{1}, W_{2}$, and

$$
2 w_{a}=\sum_{b \neq a} w_{a, b} \Longrightarrow W_{1} \subseteq W_{2}, \text { and } E\left(W_{1}\right) \subseteq E\left(W_{2}\right) .
$$

From Equation (6) with $k=3$ we get the following:

Identities 3 If $x=\{a, b, c\} \in V$,

(1) $s_{x}^{0}=\boldsymbol{\jmath}+\left(w_{a, b}+w_{a, c}+w_{b, c}\right)-\left(w_{a}+w_{b}+w_{c}\right)$;

(2) $r_{x}^{0}=-v^{x}+\boldsymbol{\jmath}+\left(w_{a, b}+w_{a, c}+w_{b, c}\right)-\left(w_{a}+w_{b}+w_{c}\right)$;

(3) $s_{x}^{1}=4 v^{x}-2\left(w_{a, b}+w_{a, c}+w_{b, c}\right)+\left(w_{a}+w_{b}+w_{c}\right)$;

(4) $r_{x}^{1}=3 v^{x}-2\left(w_{a, b}+w_{a, c}+w_{b, c}\right)+\left(w_{a}+w_{b}+w_{c}\right)$;

(5) $s_{x}^{2}=-2 v^{x}+\left(w_{a, b}+w_{a, c}+w_{b, c}\right)$;

(6) $r_{x}^{2}=-3 v^{x}+\left(w_{a, b}+w_{a, c}+w_{b, c}\right)$.

Looking at sums of the $s_{x}^{i}$ or $r_{x}^{i}$ over various ranges gives:

\section{Identities $4-i=0$}

(1) $\sum_{c \neq a, b} s_{\{a, b, c\}}^{0}=(n-4) w_{a, b}-(n-5)\left(w_{a}+w_{b}\right)+(n-5) \boldsymbol{j}$;

(2) $\sum_{c \neq a, b} r_{\{a, b, c\}}^{0}=(n-5) w_{a, b}-(n-5)\left(w_{a}+w_{b}\right)+(n-5) \boldsymbol{j}$;

(3) $\sum_{b, c \neq a} s_{\{a, b, c\}}^{0}=-\frac{1}{2}(n-3)(n-6) w_{a}+\left(\begin{array}{c}n-4 \\ 2\end{array}\right) \boldsymbol{\jmath}$;

(4) $\sum_{b, c \neq a} r_{\{a, b, c\}}^{0}=-\left(\begin{array}{c}n-4 \\ 2\end{array}\right) w_{a}+\left(\begin{array}{c}n-4 \\ 2\end{array}\right) \boldsymbol{j}$;

$-i=1$

(5) $\sum_{c \neq a, b} s_{\{a, b, c\}}^{1}=(-2 n+12) w_{a, b}+(n-7)\left(w_{a}+w_{b}\right)+3 \boldsymbol{j}$;

(6) $\sum_{c \neq a, b} r_{\{a, b, c\}}^{1}=(-2 n+11) w_{a, b}+(n-7)\left(w_{a}+w_{b}\right)+3 \boldsymbol{j}$;

(7) $\sum_{b, c \neq a} s_{\{a, b, c\}}^{1}=\frac{1}{2}\left(n^{2}-13 n+38\right) w_{a}+3(n-4) \boldsymbol{j}$;

(8) $\sum_{b, c \neq a} r_{\{a, b, c\}}^{1}=\frac{1}{2}(n-4)(n-9) w_{a}+3(n-4) \boldsymbol{j}$;

$-i=2$

(9) $\sum_{c \neq a, b} s_{\{a, b, c\}}^{2}=(n-6) w_{a, b}+2\left(w_{a}+w_{b}\right)$;

(10) $\sum_{c \neq a, b} r_{\{a, b, c\}}^{2}=(n-7) w_{a, b}+2\left(w_{a}+w_{b}\right)$;

(11) $\sum_{b, c \neq a} s_{\{a, b, c\}}^{2}=(2 n-8) w_{a}+3 \boldsymbol{j}$;

(12) $\sum_{b, c \neq a} r_{\{a, b, c\}}^{2}=(2 n-9) w_{a}+3 \boldsymbol{j}$;

To consider the possibilities of $W_{1}, W_{2}$ to be inside the dual codes of the graph, we note first the following:

Identities 5 For $a, b \in \Omega, x \in V$ :

$$
\left(w_{a}, s_{x}^{0}\right)=\left\{\begin{array}{ll}
1 & a \in x \\
\left(\begin{array}{cl}
n-4 \\
2
\end{array}\right) & a \notin x
\end{array},\left(w_{a}, r_{x}^{0}\right)=\left\{\begin{array}{ll}
0 & a \in x \\
\left(\begin{array}{c}
n-4 \\
2
\end{array}\right) & a \notin x
\end{array},\right.\right.
$$




$$
\begin{aligned}
& \left(w_{a, b}, s_{x}^{0}\right)=\left\{\begin{array}{ll}
1 & a, b \in x \\
n-5 & a \notin x, b \notin x \\
0 \quad & \text { a or } b \in x
\end{array},\left(w_{a, b}, r_{x}^{0}\right)= \begin{cases}0 & a, b \in x \\
n-5 & a \notin x, b \notin x \\
0 & \text { a or } b \in x\end{cases} \right. \\
& \left(w_{a}, s_{x}^{1}\right)=\left\{\begin{array}{ll}
1+\left(\begin{array}{c}
n-3 \\
2
\end{array}\right) & a \in x \\
3(n-4) & a \notin x
\end{array},\left(w_{a}, r_{x}^{1}\right)=\left\{\begin{array}{ll}
\left(\begin{array}{c}
n-3 \\
2
\end{array}\right) & a \in x \\
3(n-4) & a \notin x
\end{array},\right.\right. \\
& \left(w_{a, b}, s_{x}^{1}\right)=\left\{\begin{array}{ll}
1 & a, b \in x \\
3 & a \notin x, b \notin x \\
n-4 & a \text { or } b \in x
\end{array},\left(w_{a, b}, r_{x}^{1}\right)=\left\{\begin{array}{ll}
0 & a, b \in x \\
3 & a \notin x, b \notin x \\
n-4 & a \text { or } b \in x
\end{array},\right.\right. \\
& \left(w_{a}, s_{x}^{2}\right)=\left\{\begin{array}{ll}
1+2(n-3) & a \in x \\
3 & a \notin x
\end{array},\left(w_{a}, r_{x}^{2}\right)=\left\{\begin{array}{ll}
2(n-3) & a \in x \\
3 & a \notin x
\end{array},\right.\right. \\
& \left(w_{a, b}, s_{x}^{2}\right)=\left\{\begin{array}{ll}
n-2 & a, b \in x \\
0 & a \notin x, b \notin x \\
2 & \text { a or } b \in x
\end{array},\left(w_{a, b}, r_{x}^{2}\right)= \begin{cases}n-3 & a, b \in x \\
0 & a \notin x, b \notin x . \\
2 & \text { a or } b \in x\end{cases} \right.
\end{aligned}
$$

For $W_{1}, W_{2}$ :

$$
\begin{aligned}
& \left(w_{a, b}, w_{c, d}\right)=\left\{\begin{array}{rl}
n-2 & \{a, b\}=\{c, d\} \\
1 & \{c, d\} \ni \text { a or } b \\
0 & \{a, b\} \cap\{c, d\}=\emptyset
\end{array},\left(w_{a, b}, w_{c}\right)=\left\{\begin{array}{rr}
n-2 c \in\{a, b\} \\
1 & c \notin\{a, b\}
\end{array},\right.\right. \\
& \left(w_{a}, w_{b}\right)=\left\{\begin{array}{cc}
\left(\begin{array}{c}
n-1 \\
2
\end{array}\right) & a=b \\
n-2 & a \neq b
\end{array} .\right.
\end{aligned}
$$

7.2 The codes

We use the identities established in the previous subsection to obtain relationships amongst the codes. Again, throughout, $n \geq 7, k=3$, and $p \geq 5$.

From Identities 3 and since $W_{1} \subseteq W_{2}$, we have

Lemma 12 For $C=C_{i}$ or $R C_{i}$, for $i=1,2$, if $W_{2} \subseteq C$ then $C=\mathbb{F}_{p}^{V}$; if $E\left(W_{2}\right) \subseteq C$ then $\langle\boldsymbol{\jmath}\rangle^{\perp} \subseteq C$. If $\boldsymbol{\jmath} \in C_{0}$ then if $W_{2} \subseteq C_{0}$ then $C_{0}=\mathbb{F}_{p}^{V}$; if $E\left(W_{2}\right) \subseteq C_{0}$ then $\langle\boldsymbol{\jmath}\rangle^{\perp} \subseteq C_{0}$.

If $\nu \in\left\{\nu_{i}, \nu_{i}^{*} \mid 0 \leq i \leq 2\right\}$ and if $\nu \equiv 0(\bmod p)$ then the corresponding code $C \subseteq\langle\boldsymbol{\jmath}\rangle^{\perp}$.

Proof: Follows immediately from Identities 3.

Also we have

Lemma 13 1. $R C_{0} \subseteq W_{2}$;

2. if $n \equiv 5(\bmod p)$, then $W_{2}=R C_{0}$;

3. if $n \equiv 4(\bmod p)$, then $W_{1} \subseteq R C_{0}$, and $W_{1} \subseteq R C_{1}$. 
Proof: (1) From Identities 3, since $\boldsymbol{\jmath} \in W_{2}$, and $W_{1} \subseteq W_{2}$, we have $R C_{0} \subseteq W_{2}$ for all $n$, giving the first assertion.

(2) For $n \equiv 5(\bmod p)$, from Identities $4(1)$ we get $W_{2} \subseteq R C_{0}$, and hence $W_{2}=R C_{0}$.

(3) For $n \equiv 4(\bmod p)$, from Identities $4(3)$ we get $W_{1} \subseteq R C_{0}$, and from Identities $4(7)$ we get $W_{1} \subseteq R C_{1}$.

\section{Lemma 14}

(1) $W_{\Pi} \subseteq C_{0}, C_{1}, C_{2}, R C_{0}^{\perp}, R C_{1}, R C_{2}$;

(2) $W_{\Pi} \nsubseteq C_{0}^{\perp}, C_{1}^{\perp}, C_{2}^{\perp}, R C_{0}, R C_{1}^{\perp}, R C_{2}^{\perp}$.

Proof: Let $w_{\pi}$ be as defined in Equation (2). Then it can be shown directly that $\sum_{x \in X} r_{x}^{i}-\sum_{x \in X^{c}} r_{x}^{i}=\alpha_{i} w_{\pi}$, for $i=0,1,2$, where $\alpha_{0}=-1, \alpha_{1}=3, \alpha_{2}=$ -3 , and $\sum_{x \in X} s_{x}^{i}-\sum_{x \in X^{c}} s_{x}^{i}=\beta_{i} w_{\pi}$ for $i=1,2$, where $\beta_{1}=4, \beta_{2}=-2$.

That $w_{\pi} \in R C_{0}^{\perp}$, but not in $C_{0}^{\perp}, C_{1}^{\perp}, C_{2}^{\perp}, R C_{1}^{\perp}, R C_{2}^{\perp}$, can be verified directly.

To show $W_{\Pi} \nsubseteq R C_{0}$, since $R C_{0} \subseteq W_{2}=W_{\Pi}^{\perp}$ by Lemma 13 and Lemma 10, if $w_{\pi} \in R C_{0}$ then $w_{\pi} \in W_{\Pi}^{\perp}$, so $\left(w_{\pi}, w_{\pi}\right)=0$. But $\left(w_{\pi}, w_{\pi}\right)=8 \neq 0$ for $p$ odd, so we have a contradiction.

From Identities 5:

Lemma 15 1. $i=0$

$W_{1}, W_{2}, E\left(W_{2}\right) \nsubseteq R C_{0}^{\perp}$ for all $n ; E\left(W_{1}\right) \subseteq R C_{0}^{\perp}$ if $n \equiv 3,6(\bmod p)$;

$W_{1} \subseteq C_{0}^{\perp}$ for $n \equiv 4,5(\bmod p) ; W_{2} \subseteq C_{0}^{\perp}$ for $n \equiv 5(\bmod p)$;

2. $i=1$

$W_{1}, W_{2}, E\left(W_{2}\right) \nsubseteq R C_{1}^{\perp}$ for all $n ; E\left(W_{1}\right) \subseteq R C_{1}^{\perp}$ if $n^{2}-13 n+38 \equiv 0(\bmod p)$;

$W_{2}, E\left(W_{2}\right) \nsubseteq C_{1}^{\perp}$ for all $n ; W_{1} \subseteq C_{1}^{\perp}$ for $n \equiv 4(\bmod p) ; E\left(W_{1}\right) \subseteq C_{1}^{\perp}$

for $n \equiv 4,9(\bmod p)$;

3. $i=2$

$W_{1}, W_{2}, E\left(W_{2}\right) \nsubseteq R C_{2}^{\perp}$ for all $n ; E\left(W_{1}\right) \subseteq R C_{2}^{\perp}$ if $n \equiv 4(\bmod p)$;

$W_{1}, W_{2}, E\left(W_{2}\right) \nsubseteq C_{2}^{\perp}$ for all $n ; E\left(W_{1}\right) \subseteq C_{2}^{\perp}$ if $2 n \equiv 9(\bmod p)$.

Result 3 For the Kneser and Johnson graphs with $k=3$, the eigenvalues and multiplicities are as follows, using Equations (9), (10), for $A$ and $A+I$ :

- Kneser $K_{n, 3}, \nu_{0}=\left(\begin{array}{c}n-3 \\ 3\end{array}\right), n \geq 7$;

1. $\lambda_{0}=\left(\begin{array}{c}n-3 \\ 3\end{array}\right), \lambda_{0}+1=\frac{1}{6}(n-2)\left(n^{2}-10 n+27\right), m_{0}=1$;

2. $\lambda_{1}=-\left(\begin{array}{c}n-4 \\ 2\end{array}\right), \lambda_{1}+1=-\frac{1}{2}(n-3)(n-6), m_{1}=n-1$;

3. $\lambda_{2}=n-5, \lambda_{2}+1=n-4, m_{2}=\left(\begin{array}{c}n \\ 2\end{array}\right)-n$;

4. $\lambda_{3}=-1, \lambda_{3}+1=0, m_{3}=\left(\begin{array}{l}n \\ 3\end{array}\right)-\left(\begin{array}{l}n \\ 2\end{array}\right)$.

- Johnson $J(n, 3), \nu_{2}=3(n-3), n \geq 7$;

1. $\theta_{0}=3(n-3), \theta_{0}+1=3 n-8, m_{0}=1$;

2. $\theta_{1}=2 n-9, \theta_{1}+1=2 n-8, m_{1}=n-1$;

3. $\theta_{2}=n-7, \theta_{2}+1=n-6, m_{2}=\left(\begin{array}{l}n \\ 2\end{array}\right)-n$;

4. $\theta_{3}=-3, \theta_{3}+1=-2, m_{3}=\left(\begin{array}{l}n \\ 3\end{array}\right)-\left(\begin{array}{l}n \\ 2\end{array}\right)$. 
Note 5 1. Peeters in [21, p. 142] gives a formula for the $p$-rank of the Johnson graph, but there is an error in his excluded cases as for $p=5$ and $n \equiv 2(\bmod 5)$ and for $n \equiv 1(\bmod 5)$ the formulas for $A$ and $A+I$, respectively, are incorrect. Thus we will only give a value when we have all or all but one of the eigenvalues are non-zero.

2. Since $\lambda_{3}+1=0, R C_{0} \neq \mathbb{F}_{p}^{|V|}$ for all $p$.

Lemma 16 For $n \geq 7, p \geq 5$, notation as before, for $J(n, 3)$ we have:

1. if $n \not \equiv 3,7,9 / 2(\bmod p)$ then $C_{2}=\mathbb{F}_{p}^{V}$;

2. if $n \equiv 3(\bmod p)$ then $C_{2}=\langle\boldsymbol{\jmath}\rangle^{\perp}$;

3. if $n \equiv 7(\bmod p)$ and $p \neq 5$, then $\operatorname{dim}\left(C_{2}\right)=\left(\begin{array}{l}n \\ 3\end{array}\right)-\left(\begin{array}{l}n \\ 2\end{array}\right)+n$;

4. if $n \equiv 9 / 2(\bmod p)$ and $p \neq 5$, then $\operatorname{dim}\left(C_{2}\right)=\left(\begin{array}{l}n \\ 3\end{array}\right)-n+1$;

5. if $n \not \equiv 4,6,8 / 3(\bmod p)$ then $R C_{2}=\mathbb{F}_{p}^{V}$;

6. if $n \equiv 8 / 3(\bmod p), p>5$ then $R C_{2}=\langle\boldsymbol{\jmath}\rangle^{\perp}$;

7. if $n \equiv 4(\bmod p)$ then $R C_{2}^{\perp}=E\left(W_{1}\right)$;

8. if $n \equiv 6(\bmod p)$ and $p \neq 5$, then $\operatorname{dim}\left(R C_{2}\right)=\left(\begin{array}{c}n \\ 3\end{array}\right)-\left(\begin{array}{c}n \\ 2\end{array}\right)+n$. If $p=5$ then $\operatorname{dim}\left(R C_{2}\right) \geq\left(\begin{array}{l}n \\ 3\end{array}\right)-\left(\begin{array}{l}n \\ 2\end{array}\right)+n-1$.

Proof: The proof follows by determining when the number of zero eigenvalues is zero or 1, as described in Section 6.1. All cases are covered apart from when $p=5$ where the dimension of $C_{2}$ if $n \equiv 2(\bmod 5)$ and $R C_{2}$ if $n \equiv 1(\bmod 5)$ are not determined.

Lemma 17 For $n \geq 7, p \geq 5$, notation as before, for $K G_{n, 3}$ we have:

1. if $n \not \equiv 3,4,5(\bmod p)$ then $C_{0}=\mathbb{F}_{p}^{V}$; if $n \equiv 3,4,5(\bmod p)$ then $C_{0} \subseteq\langle\boldsymbol{\jmath}\rangle^{\perp}$.

2. if $n \equiv 3(\bmod p)$ then $C_{0}=\langle\boldsymbol{j}\rangle^{\perp}$;

3. if $n \equiv 0,5(\bmod p)$ then $R C_{0}=W_{2}$;

4. if $n \equiv 1,7(\bmod p), p \neq 5$ then $R C_{0}=W_{2}$.

Proof: As in Lemma 16, and using Lemma 13.

Note 6 For the reflexive case of the Kneser graphs, see also Lemma 13.

Proposition 3 If $n \equiv 6(\bmod p)$, then $R C_{1}=R C_{2}=W_{1} \oplus W_{2}^{\perp}$.

Proof: If $n \equiv 6(\bmod p)$ then $\nu_{1}^{*} \equiv 10(\bmod p)$, so $\boldsymbol{\jmath} \in R C_{1}$. From Identities $4(7), \sum_{b, c \neq a} s_{\{a, b, c\}}^{1}=\frac{1}{2}\left(n^{2}-13 n+38\right) w_{a}+3(n-4) \boldsymbol{\jmath}=-2 w_{a}+6 \boldsymbol{\jmath}$, so $W_{1} \subseteq R C_{1}$. From Lemma $14, W_{\Pi}=W_{2}^{\perp} \subseteq R C_{1}$, so $W_{1}+W_{2}^{\perp} \subseteq R C_{1}$. Now $W_{2}^{\perp} \subseteq W_{1}^{\perp}$, so $W_{1} \cap W_{2}^{\perp} \subseteq \operatorname{Hull}\left(W_{1}\right)=\{0\}$, by Proposition 2 , since, in the notation of that proposition, $n_{1} \equiv 10(\bmod p)$ and $n_{2} \equiv 4(\bmod p)$. Thus $W_{1}+W_{2}^{\perp}=W_{1} \oplus W_{2}^{\perp}$, and $W_{1} \oplus W_{2}^{\perp} \subseteq R C_{1}$.

To show that $R C_{1}=R C_{2}$, from Identities $2(3),(5), w_{x}^{1}=6 s_{x}^{1}+4 s_{x}^{2}$ and $w_{x}^{2}=4 s_{x}^{1}+6 s_{x}^{2}$, so $R C_{1}=R C_{2}$.

Finally, we need to show that $R C_{1} \subseteq W_{1} \oplus W_{2}^{\perp}$, i.e. that $s_{x}^{1} \in W_{1} \oplus W_{2}^{\perp}$ for all $x$, so we need $u \in W_{1}$ such that $s_{x}^{1}-u \in W_{2}^{\perp}$. Let $x=\{a, b, c\}$ and $u=$ 
$\sum_{d} \alpha_{d} w_{d}$. Let $N=\sum_{d} \alpha_{d}$. Recall that $\left(w_{a, b}, w_{a}\right)=n-2 \equiv 4(\bmod p)$, and $\left(w_{a, b}, w_{c}\right)=1$ for $c \neq a, b$. Now we use Identities 5 and put $\left(s_{x}^{1}-u, w_{d, e}\right)=0$ for all $d, e \in \Omega$ to obtain:

$\left(s_{x}^{1}-\sum_{d} \alpha_{d} w_{d}, w_{a, b}\right)=0 \Rightarrow 1-\sum_{d \neq a, b} \alpha_{d}-4\left(\alpha_{a}+\alpha_{b}\right)=0 \Rightarrow 1-N=3\left(\alpha_{a}+\alpha_{b}\right)$,

and similarly for $d, e \notin x$,

$$
2-N=3\left(\alpha_{a}+\alpha_{d}\right), 3-N=3\left(\alpha_{d}+\alpha_{e}\right) .
$$

This implies that $\alpha_{a}=\alpha_{b}=\alpha_{c}=\alpha$ and $\alpha_{d}=\beta$ for $d \notin x$, and so $N=3 \alpha+$ $(n-3) \beta, 1=3(\beta-\alpha)$, so $\alpha=0$ and $\beta=3^{-1}$. Thus $s_{x}^{1}-3^{-1} \sum_{d \notin x} w_{d} \in W_{2}^{\perp}$.

Recall that from Lemma $14, W_{2}^{\perp} \subseteq C_{1}, R C_{1}$ for all $p$.

Proposition 4 1. If $n \equiv 3,4(\bmod p)$ then $C_{1} \subseteq\langle\boldsymbol{J}\rangle^{\perp}$, and

(a) if $n \equiv 3(\bmod p)$ then $E\left(W_{1}\right) \subseteq W_{2}^{\perp} \subseteq C_{1}$, and if $p>5$, then $C_{1}=$ $\langle\boldsymbol{J}\rangle^{\perp}$

(b) if $n \equiv 4(\bmod p)$ then $C_{1}=C_{0}$.

2. If $n \not \equiv 3,4(\bmod p)$ then $\boldsymbol{\jmath} \in C_{1}$ and

(a) if $n \not \equiv 9(\bmod p)$ then $W_{1}+W_{2}^{\perp} \subseteq C_{1}$ and $\operatorname{dim}\left(C_{1}\right) \geq\left(\begin{array}{l}n \\ 3\end{array}\right)-\left(\begin{array}{l}n \\ 2\end{array}\right)+n-1$; if also $2 n \not \equiv 11(\bmod p)$ then $C_{1}=\mathbb{F}_{p}^{V}$;

(b) if $n \equiv 9(\bmod p)$ then $p \geq 7$, and if $p=7$ then $W_{1} \subseteq C_{1}$; if $p>7$, then $C_{1} \subseteq W_{1}^{\perp}+\langle\boldsymbol{\jmath}\rangle$.

3. For $n \not \equiv 6(\bmod p)$ :

(a) If $\nu_{1}^{*} \equiv 0(\bmod p)$, then $R C_{1} \subseteq\langle\boldsymbol{\jmath}\rangle^{\perp}$, and if $n^{2}-13 n+38 \not \equiv 0(\bmod p)$ then $R C_{1}=\langle\boldsymbol{J}\rangle^{\perp}$. If $n^{2}-13 n+38 \equiv 0(\bmod p)$ then $p=19$ and $n \equiv 0(\bmod 19), \boldsymbol{\jmath} \in R C_{1}$, and $R C_{1} \subseteq E\left(W_{1}\right)^{\perp}$.

(b) If $\nu_{1}^{*} \not \equiv 0(\bmod p)$, and $\left(n^{2}-13 n+38\right) \not \equiv 0(\bmod p)$, then $R C_{1}=\mathbb{F}_{p}^{V}$.

(c) If $\nu_{1}^{*} \neq \equiv 0(\bmod p),\left(n^{2}-13 n+38\right) \equiv 0(\bmod p)$, then $W_{2}^{\perp} \subseteq R C_{1} \subseteq$ $E\left(W_{1}\right)^{\perp}$, and $\boldsymbol{\jmath} \in R C_{1}$.

Proof: (1): If $n \equiv 3(\bmod p)$ and $p \neq 5$, then by the Identities 4(6),(8), $5 w_{a, b}-4\left(w_{a}+w_{b}\right)+3 \boldsymbol{\jmath}$ and $3 w_{a}-3 \boldsymbol{\jmath}$ are in $C_{1}$ for any $a, b \in \Omega$. The second equation gives $E\left(W_{1}\right) \subseteq C_{1}$, and then subtracting two equations of the first type gives $E\left(W_{2}\right) \subseteq C_{1}$. By Lemma $12, C_{1}=\langle\boldsymbol{\jmath}\rangle^{\perp}$. This does not work for $p=5$ although we still have $E\left(W_{1}\right) \subseteq C_{1}$. For $n \equiv 3(\bmod p), E\left(W_{1}\right) \subseteq \operatorname{Hull}\left(W_{2}\right)$ by Lemma 11.

If $n \equiv 4(\bmod p)$ then by Identities $2(2),(4), C_{0}=C_{1}$.

(2): For $n \not \equiv 3,4(\bmod p)$, if $n \not \equiv 9(\bmod p)$ then by Identities $4(8) W_{1} \subseteq C_{1}$, and $\operatorname{dim}\left(C_{1}\right) \geq \operatorname{dim}\left(W_{1}+W_{2}^{\perp}\right) \geq\left(\begin{array}{l}n \\ 3\end{array}\right)-\left(\begin{array}{c}n \\ 2\end{array}\right)+n-1$ since $\operatorname{Hull}\left(W_{2}\right) \subseteq\langle\boldsymbol{\jmath}\rangle$, by Lemma 11 . If $n \not \equiv 9(\bmod p)$ and $2 n \not \equiv 11(\bmod p)$, then similarly using also Identities 4 (8) and Lemma 12.

If $n \equiv 9(\bmod p)$, then $p \geq 7$ and by Identities $5,\left(w_{a}, r_{x}^{1}\right)=15 \neq 0$, a constant for all $a, x$, so $E\left(W_{1}\right) \subseteq C_{1}^{\perp}$. If $p=7$ then $2 n \equiv 11(\bmod p)$ so $W_{1} \subseteq C_{1}$ by Identities $4(6)$. If $p>7$ then $\left(w_{a}, \boldsymbol{\jmath}\right)=28 \neq 0$, so $\left(w_{a}, r_{x}^{1}-\frac{15}{28} \boldsymbol{\jmath}\right)=0$ for all $a, x$, so $r_{x}^{1}-\frac{15}{28} \jmath \in W_{1}^{\perp}$. 
(3): For $n \not \equiv 6(\bmod p)$, if $\nu_{1}^{*} \equiv 0(\bmod p)$ then, since $\left(s_{x}^{1}, \boldsymbol{\jmath}\right) \equiv 0(\bmod p)$, we have $R C_{1} \subseteq\langle\boldsymbol{j}\rangle^{\perp}$. If $\left(n^{2}-13 n+38\right) \not \equiv 0(\bmod p)$ then from Identities $4(7)$, $E\left(W_{1}\right) \subseteq R C_{1}$. Then Identities 4 (5) gives $E\left(W_{2}\right) \subseteq R C_{1}$, and hence by Lemma $12, R C_{1}=\langle\boldsymbol{j}\rangle^{\perp}$. If $\left(n^{2}-13 n+38\right) \equiv 0(\bmod p)$, then from Identities $5,\left(w_{a}, s_{x}^{1}\right)=\alpha$, a non-zero constant for all $a, x$, and $R C_{1} \subseteq E\left(W_{1}\right)^{\perp}$. But $\nu_{1}^{*} \equiv 0(\bmod p)$ and $\left(n^{2}-13 n+38\right) \equiv 0(\bmod p)$ implies $n \equiv 0(\bmod p)$ or $n \equiv 4(\bmod p)$. Only the first is possible with $p=19$. From Identities $4(7)$, since $n \not \equiv 4(\bmod p)$ in this case, it follows that $\boldsymbol{\jmath} \in R C_{1}$.

If $\nu_{1}^{*} \not \equiv 0(\bmod p)$ then $\boldsymbol{J} \in R C_{1}$, and from Identities $4(7)$, if $\left(n^{2}-13 n+\right.$ 38) $\not \equiv 0(\bmod p)$ then $W_{1} \subseteq R C_{1}$. From Identities $4(5)$, if $n \not \equiv 6(\bmod p)$ then $W_{2} \subseteq R C_{1}$, and hence from Lemma $12, R C_{1}=\mathbb{F}_{p}^{V}$.

If $\nu_{1}^{*} \not \equiv 0(\bmod p)$ and $\left(n^{2}-13 n+38\right) \equiv 0(\bmod p)$, then from Identities 5 , $\left(w_{a}, s_{x}^{1}\right)=\alpha$, a non-zero constant for all $a, x$. Thus $R C_{1} \subseteq E\left(W_{1}\right)^{\perp}$. From Identities $4(7)$, since $n \not \equiv 4(\bmod p)$ in this case, it follows that $\boldsymbol{\jmath} \in R C_{1}$.

Proposition 5 If $n \equiv 6(\bmod p)$ then $W_{2}=R C_{0}+W_{1}, R C_{0} \cap W_{1}=\langle\boldsymbol{\jmath}\rangle$, and $\operatorname{dim}\left(R C_{0}\right)=\left(\begin{array}{c}n-1 \\ 2\end{array}\right)$.

Proof: $R C_{0} \subseteq W_{2}$ for all $p \geq 5$ by Lemma 13. Identities 4(1) then gives $\sum_{c \neq a, b} s_{\{a, b, c\}}^{0}=2 w_{a, b}-\left(w_{a}+w_{b}\right)+\boldsymbol{\jmath}$ and (3) gives $\sum_{b, c \neq a} s_{\{a, b, c\}}^{0}=\boldsymbol{\jmath}$, and so $W_{2} \subseteq R C_{0}+W_{1}$. Since also $R C_{0}, W_{1} \subseteq W_{2}$, we have $W_{2} \stackrel{2}{=} R C_{0}+W_{1}$. Also by Lemma $15(1)$, for $n \equiv 6(\bmod p) E\left(W_{1}\right) \subseteq R C_{0}^{\perp}$, so $R C_{0} \subseteq E\left(W_{1}\right)^{\perp}$. Clearly $R C_{0} \cap W_{1} \ni \boldsymbol{\jmath}$. Suppose $v \in R C_{0} \cap W_{1}$, where $v=\sum_{i} \alpha_{i} w_{i} \in R C_{0}$. So $\left(v, w_{a}-w_{b}\right)=0$ for all $a, b \in \Omega$. Now $\left(w_{a}, w_{a}\right)=\left(\begin{array}{c}n-1 \\ 2\end{array}\right)=10$ and $\left(w_{a}, w_{b}\right)=$ $n-2=4$. So $\left(v, w_{a}-w_{b}\right)=\sum_{i} \alpha_{i}\left(w_{i}, w_{a}\right)-\sum_{i} \alpha_{i}\left(w_{i}, w_{b}\right)=10 \alpha_{a}+4 \sum_{i \neq a} \alpha_{i}-$ $10 \alpha_{b}-4 \sum_{i \neq b} \alpha_{i}=10 \alpha_{a}+4\left(\sum_{i} \alpha_{i}-\alpha_{a}\right)-10 \alpha_{b}-4\left(\sum_{i} \alpha_{i}-\alpha_{b}\right)=6 \alpha_{a}-6 \alpha_{b}=0$, so $\alpha_{a}=\alpha_{b}=\alpha$ for all $a, b \in \Omega$, and $v=3 \alpha \boldsymbol{j}$. Finally by the dimension formula we get $\operatorname{dim}\left(R C_{0}\right)=\left(\begin{array}{l}n \\ 2\end{array}\right)-n+1=\left(\begin{array}{c}n-1 \\ 2\end{array}\right)$.

Proposition 6 If $n \not \equiv 6(\bmod p)$ then

1. $E\left(W_{2}\right) \subseteq R C_{0}$;

2. if $\boldsymbol{\jmath} \in R C_{0}$, then $R C_{0}=W_{2}$, and in particular if $\nu_{0}^{*} \not \equiv 0(\bmod p)$;

3. if $\nu_{0}^{*}=\frac{1}{6}(n-2)\left(n^{2}-10 n+27\right) \equiv 0(\bmod p)$, then $n \equiv 2(\bmod p)$ or $n \equiv 5 \pm \sqrt{-2}(\bmod p)$. If $n \not \equiv 2(\bmod p)$ then $p \equiv 1,3(\bmod 8)$ and $R C_{0}=$ $E\left(W_{2}\right)$.

Proof: (1). First show that $R C_{0} \supseteq E\left(W_{2}\right)$, and first take $n \not \equiv 3,4(\bmod p)$. Identities 4(1) gives $u_{1}=(n-4) w_{a, b}-(n-5)\left(w_{a}+w_{b}\right)+(n-5) \boldsymbol{\jmath} \in R C_{0}$, and $u_{2}=(n-4) w_{a, c}-(n-5)\left(w_{a}+w_{c}\right)+(n-5) \boldsymbol{\jmath} \in R C_{0}$. We take $n \not \equiv 5(\bmod p)$ since we know $W_{2}=R C_{0}$ in this case. Thus $u_{1}-u_{2}=(n-4)\left(w_{a, b}-w_{a, c}\right)-$ $(n-5)\left(w_{b}-w_{c}\right) \in R C_{0}$. From $4(3), u_{3}=-\frac{1}{2}(n-3)(n-6) w_{b}+\left(\begin{array}{c}n-4 \\ 2\end{array}\right) \boldsymbol{\jmath} \in R C_{0}$ and $u_{4}=-\frac{1}{2}(n-3)(n-6) w_{c}+\left(\begin{array}{c}n-4 \\ 2\end{array}\right) \boldsymbol{\jmath} \in R C_{0}$. Thus $u_{3}-u_{4}=-\frac{1}{2}(n-3)(n-$ $6)\left(w_{b}-w_{c}\right) \in R C_{0}$, and since also $n \not \equiv 3(\bmod p)$, we have $E\left(W_{1}\right) \subseteq R C_{0}$ and then from the above that $E\left(W_{2}\right) \subseteq R C_{0}$ for $n \not \equiv 3,4,6(\bmod p)$.

Now suppose $n \equiv 3(\bmod p)$. Then $\nu_{0}^{*} \neq 0$, so $\boldsymbol{\jmath} \in R C_{0}$. From Identities 4(1), we have $-w_{a, b}+2\left(w_{a}+w_{b}\right),-w_{a, c}+2\left(w_{a}+w_{c}\right),-w_{b, c}+2\left(w_{b}+w_{c}\right) \in$ 
$R C_{0}$, and summing gives $-\left(w_{a, b}+w_{a, c}+w_{b, c}\right)+4\left(w_{a}+w_{b}+w_{c}\right) \in R C_{0}$. From Identities 3 , we see that $4\left(w_{a, b}+w_{a, c}+w_{b, c}\right)-4\left(w_{a}+w_{b}+w_{c}\right) \in R C_{0}$, and summing with the previous element gives $\left(w_{a, b}+w_{a, c}+w_{b, c}\right) \in R C_{0}$ and $\left(w_{a}+w_{b}+w_{c}\right) \in R C_{0}$. Now $R C_{0} \ni-w_{a, b}+2\left(w_{a}+w_{b}\right)=-w_{a, b}+2\left(w_{a}+w_{b}+\right.$ $\left.w_{c}\right)-2 w_{c}$, and hence $w_{a, b}+2 w_{c} \in R C_{0}$ for all $a, b, c$ and subtracting shows that $E\left(W_{2}\right) \subseteq R C_{0}$.

Now suppose $n \equiv 4(\bmod p)$. Then $\nu_{0}^{*} \neq 0$, so $\boldsymbol{\jmath} \in R C_{0}$, and $W_{1} \subseteq R C_{0}$ by Identities 4(3). From Identities 3, we see that $\left(w_{a, b}+w_{a, c}+w_{b, c}\right) \in R C_{0}$ for all $a, b, c \in \Omega$. In particular we have $\left(w_{a, b}+w_{a, d}+w_{b, d}\right),\left(w_{a, d}+w_{a, c}+w_{d, c}\right) \in R C_{0}$, and subtracting gives $\left(w_{a, b}-w_{a, c}\right)+\left(w_{d, b}-w_{d, c}\right) \in R C_{0}$. By symmetry we also have $\left(w_{c, a}-w_{c, d}\right)+\left(w_{b, a}-w_{b, d}\right) \in R C_{0}$. Summing these two last elements gives $2\left(w_{a, b}-w_{c, d}\right) \in R C_{0}$ and hence $E\left(W_{2}\right) \subseteq R C_{0}$.

(2). Suppose that $\boldsymbol{\jmath} \in R C_{0}$. Since $3 \boldsymbol{\jmath}=\sum_{a, b} w_{a, b}=\sum_{a, b}\left(w_{a, b}-w_{c, d}\right)+\left(\begin{array}{l}n \\ 2\end{array}\right) w_{c, d}$, if $n \not \equiv 0,1(\bmod p), w_{c, d} \in R C_{0}$. However, for $n \equiv 0,1(\bmod p)$ we have $R C_{0}=$ $W_{2}$ by Lemma 17 , unless $p=5$ and $n \equiv 1(\bmod 5)$. But we have excluded this case because $1 \equiv 6(\bmod 5)$. Thus the result follows since $\nu_{0}^{*} \boldsymbol{J}=\sum_{x \in V} s_{x}^{0}$.

(3). If $\nu_{0}^{*} \equiv 0(\bmod p)$ and $n \not \equiv 2(\bmod p)$ then $n \equiv 5 \pm \sqrt{-2}(\bmod p)$. Now it can be proved that -2 is a square in $\mathbb{F}_{p}$ for prime $p \geq 5$ if and only if $p \equiv 1,3(\bmod 8):$ see $[2$, Lemma 2.10 .1$]$ where the proof can be modified easily to the case of -2 . If $\nu_{0}^{*} \equiv 0(\bmod p)$ then $R C_{0} \subset\langle\boldsymbol{\jmath}\rangle^{\perp}$, so if $R C_{0}=W_{2}$ then $W_{2} \subset\langle\boldsymbol{\jmath}\rangle^{\perp}$, so $n \equiv 2(\bmod p)$. Thus if $n \not \equiv 2(\bmod p)$ and $\nu_{0}^{*} \equiv 0(\bmod p)$ then $R C_{0}=E\left(W_{2}\right)$.

For design theory notation see [2, Chapter 1], and for a discussion of Steiner triple systems see [2, Chapter 8].

Proposition 7 For all primes $p$, if $n \geq 7$ and $n \equiv 1,3(\bmod 6)$ then $\frac{1}{6}\left(n^{2}-\right.$ $10 n+27) \boldsymbol{\jmath} \in R C_{0}$. If also $n \equiv 2(\bmod p)$ and $p \geq 5$, then $R C_{0}=W_{2}$ unless, possibly, $p=11$.

Proof: By $[17,20]$, a Steiner triple system on $n$ points, i.e. a $2-(n, 3,1)$ design, exists if and only if $n \equiv 1,3(\bmod 6)$. Let $\mathcal{S}$ be a Steiner triple system on $n$ points with block set $\mathcal{B}$. Then if $\Omega=\{1, \ldots, n\}, \mathcal{B} \subset \Omega^{\{3\}}=V$, and the number $r$ of blocks through a point is $r=\frac{1}{2}(n-1)$. Thus $b=|\mathcal{B}|=\frac{1}{6} n(n-1)$.

Let $w=\sum_{x \in \mathcal{B}} s_{x}^{0}$. For $x \in \mathcal{B}, x$ meets $3(r-1)=\frac{3}{2}(n-3)$ other blocks, so $x$ is disjoint from, or equal to, $b-3(r-1)=b-\frac{3}{2}(n-3)=\frac{1}{6}\left(n^{2}-10 n+27\right)$ blocks. Thus for $x \in \mathcal{B}, w(x)=\frac{1}{6}\left(n^{2}-10 n+27\right)$.

For $x \in \Omega^{\{3\}} \backslash \mathcal{B}$, we have $x=\{a, b, c\}$ is not a block of $\mathcal{S}$, and blocks in $\mathcal{B}$ can meet $x$ in only one element, so the number of blocks meeting $x$ is $3 r-3$, since the blocks $a b, a c$ and $b c$ of $\mathcal{B}$ are counted twice. Thus the number not meeting $x$ is $b-(3 r-3)=\frac{1}{6}\left(n^{2}-10 n+27\right)$, as above, so for $x \notin \mathcal{B}$, we have $w(x)=\frac{1}{6}\left(n^{2}-10 n+27\right)$, and so $w=\frac{1}{6}\left(n^{2}-10 n+27\right) \boldsymbol{\jmath}$, as asserted.

Suppose $n=2+k p$. Then $\frac{1}{6}\left(n^{2}-10 n+27\right)=\frac{1}{6}\left((2+k p)^{2}-10(2+k p)+27\right)=$ $\frac{1}{6}\left((k p)^{2}-6(k p)+11\right)=\frac{1}{6} 11$, which is non-zero if $p \neq 11$.

Lemma 18 If $p=5, n \geq 7, n \equiv 2(\bmod 5)$, then $E\left(C_{2}\right)=W_{1}+W_{2}^{\perp}$, and $\operatorname{dim}\left(C_{2}\right)=\left(\begin{array}{l}n \\ 3\end{array}\right)-\left(\begin{array}{l}n \\ 2\end{array}\right)+n$. 
Proof: By Identities 4 (10), $w_{a}+w_{b} \in C_{2}$ for all $a, b \in \Omega$, and hence $W_{1} \subseteq$ $C_{2}$. By Lemmas $10,14, W_{\Pi}=W_{2}^{\perp} \subseteq C_{2}$. It is quite direct to show that $W_{1} \cap W_{2}^{\perp}=\langle\boldsymbol{\jmath}\rangle$, and thus $\operatorname{dim}\left(W_{1}+W_{2}^{\perp}\right)=\left(\begin{array}{l}n \\ 3\end{array}\right)-\left(\begin{array}{l}n \\ 2\end{array}\right)+n-1$. We show that $E\left(C_{2}\right)=W_{1}+W_{2}^{\perp}$ by showing that for each pair $x, y \in V$, there is an element $w=\sum_{i=1}^{n} \alpha_{i} w_{i}$ such that $r_{x}^{2}-r_{y}^{2}-w \in W_{2}^{\perp}$. There are three cases for $x, y$ to be considered and they are covered by the following: (1) $r_{\{1,2,3\}}^{2}-r_{\{1,2,4\}}^{2}+2\left(w_{3}-w_{4}\right) ;(2) r_{\{1,2,3\}}^{2}-r_{\{1,4,5\}}^{2}-3\left(w_{2}+w_{3}\right)-2\left(w_{4}+w_{5}\right)$; (3) $r_{\{1,2,3\}}^{2}-r_{\{4,5,6\}}^{2}+2\left(w_{1}+w_{2}+w_{3}\right)-2\left(w_{4}+w_{5}+w_{6}\right)$.

Thus $E\left(C_{2}\right) \subseteq W_{1}+W_{2}^{\perp} \subseteq C_{2}$. Since $W_{1}+W_{2}^{\perp} \subseteq\langle\boldsymbol{\jmath}\rangle^{\perp}$, but $C_{2} \not \subset\langle\boldsymbol{\jmath}\rangle^{\perp}$, we have $W_{1}+W_{2}^{\perp}=E\left(C_{2}\right)$, and thus $\operatorname{dim}\left(C_{2}\right)=\left(\begin{array}{l}n \\ 3\end{array}\right)-\left(\begin{array}{l}n \\ 2\end{array}\right)+n$.

\section{Summary for codes from $\Gamma(n, 3, r)$ and $R \Gamma(n, 3, r), n \geq 7, p \geq 5$}

We summarize the results for the codes $C_{i}$ and $R C_{i}$ from $\Gamma(n, 3, r)$ and $R \Gamma(n, 3, r)$, respectively, for $p \geq 5$ that we have established in Section 7 . Note that there are open questions remaining. Take $n \geq 7, p \geq 5$ in all the following.

\section{$8.1 C_{0}$}

$\nu_{0}=\left(\begin{array}{c}n-3 \\ 3\end{array}\right) ; \forall p, W_{2}^{\perp} \subseteq C_{0}, W_{2} \nsupseteq C_{0}$ (Lemma 14)

1. $n \not \equiv 3,4,5(\bmod p) \Longrightarrow C_{0}=\mathbb{F}_{p}^{V}($ Lemma $17(1))$

2. $n \equiv 3(\bmod p) \Longrightarrow C_{0}=\langle\boldsymbol{\jmath}\rangle^{\perp}($ Lemma $17(2))$

3. $n \equiv 4(\bmod p) \Longrightarrow C_{0}=W_{1}^{\perp}\left(\right.$ Lemma 15 (1) gives $C_{0} \subseteq W_{1}^{\perp}$ so $\operatorname{dim}\left(C_{0}\right) \leq\left(\begin{array}{l}n \\ 3\end{array}\right)-n$. Eigenvalues for Kneser gives $\lambda_{0}=\lambda_{1}=0$, and the other two non-zero, so $\operatorname{dim}\left(C_{0}\right) \geq\left(\begin{array}{l}n \\ 3\end{array}\right)-\left(m_{0}+m_{1}\right)=\left(\begin{array}{l}n \\ 3\end{array}\right)-n$. Thus $\operatorname{dim}\left(C_{0}\right)=\left(\begin{array}{l}n \\ 3\end{array}\right)-n$ and $\left.C_{0}=W_{1}^{\perp}\right)$

4. $n \equiv 5(\bmod p) \Longrightarrow C_{0}=W_{2}^{\perp}\left(\right.$ Lemma 14 (1) gives $W_{2}^{\perp} \subseteq C_{0}$, Lemma 15 (1) gives $C_{0} \subseteq W_{2}^{\perp}$, hence equality)

\begin{tabular}{||l||l|l|l|l||}
\hline \hline$n$ cong & $\not \equiv 3,4,5$ & $\equiv 3$ & $\equiv 4$ & $\equiv 5$ \\
\hline \hline$p \geq 5$ & & & & \\
\hline$\nu$ & $\not \equiv 0$ & $\equiv 0$ & $\equiv 0$ & $\equiv 0$ \\
\hline$C_{0}$ & $\mathbb{F}_{p}^{V}$ & $\langle\boldsymbol{\jmath}\rangle^{\perp}$ & $W_{1}^{\perp}$ & $W_{2}^{\perp}$ \\
\hline \hline
\end{tabular}

Table $1 C_{0}, p \geq 5$, congruences modulo $p$ 
$8.2 R C_{0}$

$\nu_{0}^{*}=\nu_{0}+1=\left(\begin{array}{c}n-3 \\ 3\end{array}\right)+1=\frac{1}{6}(n-2)\left(n^{2}-10 n+27\right) ; \forall p, R C_{0} \subseteq W_{2}, W_{2}^{\perp} \nsubseteq R C_{0}$ (Lemma 14)

1. $n \equiv 6(\bmod p) \Longrightarrow W_{2}=R C_{0}+W_{1}, R C_{0} \cap W_{1}=\langle\boldsymbol{\jmath}\rangle, \operatorname{dim}\left(R C_{0}\right)=\left(\begin{array}{c}n-1 \\ 2\end{array}\right)$ (Proposition 5)

2. $n \not \equiv 6(\bmod p) \Longrightarrow E\left(W_{2}\right) \subseteq R C_{0} \subseteq W_{2}$ (Proposition 6 and Lemma 13)

(a) $\nu_{0}^{*} \not \equiv 0(\bmod p) \Longrightarrow R C_{0}=W_{2}$ (Proposition 6$)$

(b) $\nu_{0}^{*} \equiv 0(\bmod p), n \not \equiv 2(\bmod p) \Longrightarrow n \equiv 5 \pm \sqrt{-2}(\bmod p)$, for $p \equiv 1,3(\bmod 8) \Longrightarrow R C_{0}=E\left(W_{2}\right)$ (Proposition 6$)$

(c) $n \equiv 2(\bmod p), n \equiv 1,3(\bmod 6), p \neq 11 \Longrightarrow R C_{0}=W_{2}$ (Proposition 7)

\begin{tabular}{||l||l|l|l|l|l||}
\hline \hline$n$ cong & $\equiv 6$ & $\begin{array}{l}\equiv 2, \\
\equiv 1,3(\bmod 6)\end{array}$ & $\begin{array}{l}\not \equiv 6 \\
\equiv 2,6, \\
\equiv 5 \pm \sqrt{-2}\end{array}$ & $\begin{array}{l}\equiv 2, \\
\equiv \equiv 1,3(\bmod 6)\end{array}$ \\
\hline \hline$p \geq 5$ & & $\neq 11$ & & & \\
\hline$\nu^{*}$ & $\not \equiv 0$ & $\equiv 0$ & $\not \equiv 0$ & $\equiv 0$ & $\equiv 0$ \\
\hline$R C_{0}$ & $W_{2}=R C_{0}+W_{1}$ & $W_{2}$ & $W_{2}$ & $E\left(W_{2}\right)$ & $E\left(W_{2}\right) \subseteq R C_{0} \subseteq W_{2}$ \\
\hline \hline
\end{tabular}

Table $2 R C_{0}, p \geq 5$, congruences modulo $p$

$8.3 C_{1}$

$\nu_{1}=3\left(\begin{array}{c}n-3 \\ 2\end{array}\right) ; \forall p, W_{2}^{\perp} \subseteq C_{1}, W_{2} \nsupseteq C_{1}($ Lemma 14)

1. $n \equiv 3(\bmod p) \Longrightarrow E\left(W_{1}\right) \subseteq C_{1} \subseteq\langle\boldsymbol{\jmath}\rangle^{\perp}$ and for $p>5, C_{1}=\langle\boldsymbol{\jmath}\rangle^{\perp}$ (Proposition 4)

2. $n \equiv 4(\bmod p) \Longrightarrow C_{1}=W_{1}^{\perp}$ (Identities 2 gives $C_{1}=C_{0}$, and $C_{0}=W_{1}^{\perp}$ by Subsection 8.1)

3. if $n \equiv 9(\bmod p)$ then $p \geq 7$, and if $p=7$ then $W_{1} \subseteq C_{1}$; if $p>7$, then $C_{1} \subseteq W_{1}^{\perp}+\langle\boldsymbol{j}\rangle$. (Proposition 4)

4. $n \not \equiv 3,4,9(\bmod p) \Longrightarrow W_{1} \subseteq C_{1}, \operatorname{dim}\left(C_{1}\right) \geq \operatorname{dim}\left(W_{2}^{\perp}\right)+n-1$ (Proposition 4)

5. $n \not \equiv 3,4,9(\bmod p), 2 n \not \equiv 11(\bmod p) \Longrightarrow C_{1}=\mathbb{F}_{p}^{V}($ Proposition 4$)$

\section{$8.4 R C_{1}$}

$\nu_{1}^{*}=\nu_{1}+1=3\left(\begin{array}{c}n-3 \\ 2\end{array}\right)+1=\frac{1}{2}\left(3 n^{2}-21 n+38\right) ; \forall p, W_{2}^{\perp} \subseteq R C_{1}, W_{2} \nsupseteq R C_{1}$ (Lemma 14)

1. $n \equiv 6(\bmod p) \Longrightarrow R C_{1}=W_{1} \oplus W_{2}^{\perp}\left(R C_{1}=R C_{2}\right.$ by Identities $2(3),(5)$, so see 11.6 below) 


\begin{tabular}{||l||l|l|l|l|l|l|l||}
\hline \hline$n$ cong & $\equiv 3$ & $\equiv 3$ & $\equiv 4$ & $\equiv 9$ & $\equiv 9$ & $\not \equiv 3,4,9$ & $\begin{array}{l}\not \equiv 3,4, \\
9, \frac{11}{2}\end{array}$ \\
\hline \hline$p \geq 5$ & 5 & $>5$ & & 7 & $>7$ & & \\
\hline$\nu$ & $\equiv 0$ & $\equiv 0$ & $\equiv 0$ & $\not \equiv 0$ & $\not \equiv 0$ & $\not 00$ & $\not \equiv 0$ \\
\hline$C_{1} \supseteq W_{2}^{\perp}$ & $E\left(W_{1}\right) \subseteq C_{1}$ & $\langle\boldsymbol{\jmath}\rangle^{\perp}$ & $W_{1}^{\perp}$ & $\supseteq W_{1}+W_{2}^{\perp}$ & $\subseteq\left\langle W_{1}^{\perp}, \boldsymbol{\jmath}\right\rangle$ & $\supseteq W_{1}+W_{2}^{\perp}$ & $\mathbb{F}_{p}^{V}$ \\
\hline \hline
\end{tabular}

Table $3 C_{1}, p \geq 5$, congruences modulo $p$

2. $n \not \equiv 6(\bmod p), \nu_{1}^{*} \equiv 0(\bmod p) \Longrightarrow R C_{1} \subseteq\langle\boldsymbol{\jmath}\rangle^{\perp},\left(n^{2}-13 n+38\right) \not \equiv 0(\bmod p)$ $\Longrightarrow R C_{1}=\langle\boldsymbol{\jmath}\rangle^{\perp} ;\left(n^{2}-13 n+38\right) \equiv 0(\bmod p) \Longrightarrow n \equiv 0(\bmod 19), p=$ $19 \Longrightarrow R C_{1} \subseteq E\left(W_{1}\right)^{\perp}$ (Proposition 4)

3. $n \not \equiv 6(\bmod p), \nu_{1}^{*} \not \equiv 0(\bmod p),\left(n^{2}-13 n+38\right) \not \equiv 0(\bmod p) \Longrightarrow R C_{1}=$ $\mathbb{F}_{p}^{V}$ (Proposition 4)

4. $n \not \equiv 6(\bmod p), \nu_{1}^{*} \not \equiv 0(\bmod p),\left(n^{2}-13 n+38\right) \equiv 0(\bmod p) \Longrightarrow W_{2}^{\perp} \subseteq$ $R C_{1} \subseteq E\left(W_{1}\right)^{\perp}$ (Proposition 4$)$.

\begin{tabular}{||l||l|l|l|l|l||}
\hline \hline$n$ cong & $\equiv 6$ & $\begin{array}{l}\not \equiv 6 ; \\
h(n) \not \equiv 0\end{array}$ & $\begin{array}{l}\not \equiv 6 ; \\
h(n) \equiv 0\end{array}$ & $\begin{array}{l}\not \equiv 6 ; \\
h(n) \not \equiv 0\end{array}$ & $\begin{array}{l}\not \equiv 6 ; \\
h(n) \equiv 0\end{array}$ \\
\hline \hline$p \geq 5$ & $5,>5$ & & 19 & & \\
\hline$\nu^{*}$ & $\equiv 0, \not \equiv 0$ & $\equiv 0$ & $\equiv 0$ & $\not \equiv 0$ & $\not \equiv 0$ \\
\hline$R C_{1} \supseteq W_{2}^{\perp}$ & $W_{1} \oplus W_{2}^{\perp}$ & $\langle\boldsymbol{\jmath}\rangle^{\perp}$ & $\subseteq E\left(W_{1}\right)^{\perp}$ & $\mathbb{F}_{p}^{V}$ & $\subseteq E\left(W_{1}\right)^{\perp}$ \\
\hline \hline
\end{tabular}

Table $4 R C_{1}, p \geq 5$, congruences modulo $p, h(n)=n^{2}-13 n+38$

$8.5 C_{2}$

$\nu_{2}=3(n-3) ; \forall p, W_{2}^{\perp} \subseteq C_{2}, W_{2} \nsupseteq C_{2}$ (Lemma 14)

1. $n \not \equiv 3,7,9 / 2(\bmod p) \Longrightarrow C_{2}=\mathbb{F}_{p}^{V}($ Lemma 16)

2. $n \equiv 3(\bmod p) \Longrightarrow C_{2}=\langle\boldsymbol{\jmath}\rangle^{\perp}($ Lemma 16$)$

3. $n \equiv 7(\bmod p), p \neq 5 \Longrightarrow \operatorname{dim}\left(C_{2}\right)=\left(\begin{array}{l}n \\ 3\end{array}\right)-\left(\begin{array}{l}n \\ 2\end{array}\right)+n, C_{2}=W_{1} \oplus W_{2}^{\perp}$ (Lemma 16 and $\boldsymbol{\jmath} \in C_{2} \Longrightarrow W_{1} \subseteq C_{2}$ by Identities 4 (12), $W_{1} \cap W_{2}^{\perp} \subseteq$ $\operatorname{Hull}\left(W_{2}\right)=\{0\}$ by Lemma 11)

4. $n \equiv 9 / 2(\bmod p), p \neq 5 \Longrightarrow \operatorname{dim}\left(C_{2}\right)=\left(\begin{array}{l}n \\ 3\end{array}\right)-n+1, C_{2}=E\left(W_{1}\right)^{\perp}$ (Lemma 16, and by Identities 5, $C_{2} \subseteq E\left(W_{1}\right)^{\perp}$, and $\operatorname{dim}\left(E\left(W_{1}\right)^{\perp}\right)=$ $\left.\left(\begin{array}{l}n \\ 3\end{array}\right)-n+1\right)$

5. $n \equiv 2(\bmod p), p=5 \Longrightarrow E\left(C_{2}\right)=W_{1}+W_{2}^{\perp}, \operatorname{dim}\left(C_{2}\right)=\left(\begin{array}{l}n \\ 3\end{array}\right)-\left(\begin{array}{l}n \\ 2\end{array}\right)+n$

(Lemma 18)

$8.6 R C_{2}$

$\nu_{2}^{*}=\nu_{2}+1=3(n-3)+1 ; \forall p, W_{2}^{\perp} \subseteq R C_{2}, W_{2} \nsupseteq R C_{2}$ (Lemma 14) 


\begin{tabular}{||l||l|l|l|l|l||}
\hline \hline$n$ cong & $\not \equiv 3,7, \frac{9}{2}$ & $\equiv 3$ & $\equiv 7$ & $\equiv \frac{9}{2}$ & $\equiv 2$ \\
\hline \hline$p \geq 5$ & & & $>5$ & $>5$ & 5 \\
\hline$\nu$ & $\not \equiv 0$ & $\equiv 0$ & $\not \equiv 0$ & $\not \equiv 0$ & $\not \equiv 0$ \\
\hline$C_{2}$ & $\mathbb{F}_{p}^{V}$ & $\langle\boldsymbol{\jmath}\rangle^{\perp}$ & $W_{1} \oplus W_{2}^{\perp}$ & $E\left(W_{1}\right)^{\perp}$ & $E\left(C_{2}\right)=W_{1}+W_{2}^{\perp}$ \\
\hline
\end{tabular}

Table $5 C_{2}, p \geq 5$, congruences modulo $p$

1. if $n \not \equiv 4,6,8 / 3(\bmod p) \Longrightarrow R C_{2}=\mathbb{F}_{p}^{V}$ (Lemma 16)

2. if $n \equiv 8 / 3(\bmod p), p>5 \Longrightarrow R C_{2}=\langle\boldsymbol{\jmath}\rangle^{\perp}($ Lemma 16)

3. if $n \equiv 4(\bmod p) \Longrightarrow R C_{2}=E\left(W_{1}\right)^{\perp}($ Lemma 16)

4. if $n \equiv 6(\bmod p) \Longrightarrow \operatorname{dim}\left(R C_{2}\right)=\left(\begin{array}{l}n \\ 3\end{array}\right)-\left(\begin{array}{l}n \\ 2\end{array}\right)+n, R C_{2}=W_{1} \oplus W_{2}^{\perp}$ (for $p \neq 5$ : Lemma 16 , and $\boldsymbol{\jmath} \in R C_{2} \Longrightarrow W_{1} \subseteq R C_{2}$ by Identities 4 (11), $W_{1} \cap W_{2}^{\perp} \subseteq \operatorname{Hull}\left(W_{2}\right)=\{0\}$ by Lemma 11 . For $p=5$ : by Lemma 16 , $\operatorname{dim}\left(R C_{2}\right) \geq\left(\begin{array}{l}n \\ 3\end{array}\right)-m_{0}-m_{2}=\left(\begin{array}{l}n \\ 3\end{array}\right)-\left(\begin{array}{l}n \\ 2\end{array}\right)+n-1$; by Identities $4(9)$, $W_{1} \subseteq R C_{2}$, so $W_{1} \oplus W_{2}^{\perp} \subseteq R C_{2}$. Since $s_{\{a, b, c\}}^{2}-2 \boldsymbol{\jmath}+w_{a}+w_{b}+w_{c} \in W_{2}^{\perp}$, equality follows)

\begin{tabular}{||l||l|l|l|l|l||}
\hline \hline$n$ cong & $\not \equiv 4,6, \frac{8}{3}$ & $\equiv \frac{8}{3}$ & $\equiv 4$ & $\equiv 6$ & $\equiv 6$ \\
\hline \hline$p \geq 5$ & & $>5$ & & $>5$ & 5 \\
\hline$\nu^{*}$ & $\not \equiv 0$ & $\equiv 0$ & $\not \equiv 0$ & $\not \equiv 0$ & $\equiv 0$ \\
\hline$R C_{2}$ & $\mathbb{F}_{p}^{V}$ & $\langle\boldsymbol{\jmath}\rangle^{\perp}$ & $E\left(W_{1}\right)^{\perp}$ & $W_{1} \oplus W_{2}^{\perp}$ & $W_{1} \oplus W_{2}^{\perp}$ \\
\hline \hline
\end{tabular}

Table $6 R C_{2}, p \geq 5$, congruences modulo $p$

According to Magma, the unsolved cases for $k=3, p \geq 5$ :

$8.2(2)(\mathrm{c}): R C_{0}=W_{2}$ unless $p=11$ when $R C_{0}=E\left(W_{2}\right) ; 8.3$ (1): for $p=5$, $\operatorname{dim}\left(C_{1}\right)=\operatorname{dim}\left(W_{2}^{\perp}\right)+n-1 ; 8.3(3)$ : for $p=7, \operatorname{dim}\left(C_{1}\right)=\operatorname{dim}\left(W_{2}^{\perp}\right)+n$; $p>7, C_{1}=W_{1}^{\perp}+\langle\boldsymbol{\jmath}\rangle ;$ 8.3.(4): $\operatorname{dim}\left(C_{1}\right)=\operatorname{dim}\left(W_{2}^{\perp}\right)+n-1 ; 8.4$ (2): if $p=19, n \equiv 0(\bmod 19)$ then $R C_{1}=E\left(W_{1}\right)^{\perp} ; 8.4(4): R C_{1}=E\left(W_{1}\right)^{\perp}$.

8.7 Summary for codes from $\Gamma(n, 3, r)$ for $p=2,3,5,7$

Tables 7,8 summarize the results from $[14,15,12,11]$ for $\Gamma(n, 3, r)$ and $R \Gamma(n, 3, r)$ for $p=2,3$, respectively. Tables 9,10 summarize what we have shown in Tables 1 to 6 for $p=5,7$, respectively. In the tables, $H(C)=\operatorname{Hull}(C)$.

\section{Conclusion}

It can be shown by similar methods that for $\Gamma(n, 2, r)$ and $R \Gamma(n, 2, r), r=0,1$, $C_{r}, R C_{r}$ are always one of

$$
\langle\boldsymbol{\jmath}\rangle^{\perp}, \mathbb{F}_{p}^{V}, W_{1}, E\left(W_{1}\right), W_{1}^{\perp}, E\left(W_{1}\right)^{\perp},\left\langle\boldsymbol{\jmath}, W_{1}\right\rangle,\left\langle\boldsymbol{\jmath}, E\left(W_{1}\right)\right\rangle, W_{\Pi}, W_{\Pi}^{\perp} .
$$




\begin{tabular}{||l||l|l|l|l|l|l||}
\hline \hline$n$ cong & $C_{0}$ & $R C_{0}$ & $C_{1}$ & $R C_{1}$ & $C_{2}$ & $R C_{2}$ \\
\hline \hline 0 & $W_{1}^{\perp}$ & $W_{1}+H\left(W_{2}\right)$ & $W_{1}^{\perp}$ & $W_{1}$ & $\mathbb{F}_{2}^{V}$ & $H\left(W_{2}\right)$ \\
\hline 1 & $W_{1}^{\perp} \cap W_{2}^{\perp}$ & $W_{1}+W_{2}$ & $E\left(W_{1}\right)^{\perp}$ & $W_{1}$ & $W_{2}^{\perp}$ & $W_{2}$ \\
\hline 2 & $\mathbb{F}_{2}^{V}$ & $\subset W_{1}+H\left(W_{2}\right)$ & $\mathbb{F}_{2}^{V}$ & $W_{1}$ & $\mathbb{F}_{2}^{V}$ & $H\left(W_{2}\right)$ \\
\hline 3 & $W_{2}^{\perp}$ & $W_{1}+W_{2}$ & $\langle\boldsymbol{\jmath}\rangle^{\perp}$ & $W_{1}$ & $W_{2}^{\perp}$ & $W_{2}$ \\
\hline \hline
\end{tabular}

Table $7 \Gamma(n, 3, r), R \Gamma(n, 3, r), p=2$, congruences modulo 4

\begin{tabular}{||l||l|l|l|l|l|l||}
\hline \hline$n$ cong & $C_{0}$ & $R C_{0}$ & $C_{1}$ & $R C_{1}$ & $C_{2}$ & $R C_{2}$ \\
\hline \hline 0,0 & $\mathbb{F}_{3}^{V}$ & $\subset W_{2}+\langle\boldsymbol{\jmath}\rangle$ & $E\left(W_{2}\right)$ & $R C_{0}^{\perp}+W_{2}^{\perp}$ & $E\left(W_{2}\right)$ & $R C_{1}$ \\
\hline 0,3 & $\langle\boldsymbol{\jmath}\rangle^{\perp}$ & $\subset W_{2}+\langle\boldsymbol{\jmath}\rangle$ & $E\left(W_{2}\right)$ & $R C_{0}^{\perp}+W_{2}^{\perp}$ & $E\left(W_{2}\right)$ & $R C_{1}$ \\
\hline 0,6 & $\mathbb{F}_{3}^{V}$ & $\subset W_{2}+\langle\boldsymbol{\jmath}\rangle$ & $E\left(W_{2}\right)$ & $R C_{0}^{\perp}+W_{2}^{\perp}$ & $E\left(W_{2}\right)$ & $R C_{1}$ \\
\hline 1,1 & $W_{1}^{\perp}$ & $E\left(W_{2}\right)+\langle\boldsymbol{\jmath}\rangle$ & $H\left(W_{2}\right)$ & $\mathbb{F}_{3}^{V}$ & $E\left(W_{2}\right)$ & $W_{1}^{\perp}$ \\
\hline 1,4 & $W_{1}^{\perp}=C_{0}+\langle\boldsymbol{\jmath}\rangle$ & $E\left(W_{2}\right)+\langle\boldsymbol{\jmath}\rangle$ & $H\left(W_{2}\right)$ & $\mathbb{F}_{3}^{V}$ & $E\left(W_{2}\right)$ & $W_{1}^{\perp}$ \\
\hline 1,7 & $W_{1}^{\perp}$ & $E\left(W_{2}\right)+\langle\boldsymbol{\jmath}\rangle$ & $H_{2}\left(W_{2}\right)$ & $\mathbb{F}_{3}^{V}$ & $E\left(W_{2}\right)$ & $W_{1}^{\perp}$ \\
\hline 2,2 & $W_{2}^{\perp}$ & $W_{2}+\langle\boldsymbol{\jmath}\rangle$ & $W_{2}$ & $\mathbb{F}_{3}^{V}$ & $W_{2}$ & $\mathbb{F}_{3}^{V}$ \\
\hline 2,5 & $\left(W_{2}+\langle\boldsymbol{\jmath}\rangle\right)^{\perp}$ & $W_{2}+\langle\boldsymbol{\jmath}\rangle$ & $W_{2}$ & $\mathbb{F}_{3}^{V}$ & $W_{2}$ & $\mathbb{F}_{3}^{V}$ \\
\hline 2,8 & $W_{2}^{\perp}$ & $W_{2}+\langle\boldsymbol{\jmath}\rangle$ & $W_{2}$ & $\mathbb{F}_{3}^{V}$ & $W_{2}$ & $\mathbb{F}_{3}^{V}$ \\
\hline \hline
\end{tabular}

Table $8 \Gamma(n, 3, r), R \Gamma(n, 3, r), p=3$, congruences modulo 3,9

\begin{tabular}{|l||l|l||l|l||l|l|}
\hline \hline$n$ cong & $C_{0}$ & $R C_{0}$ & $C_{1}$ & $R C_{1}$ & $C_{2}$ & $R C_{2}$ \\
\hline \hline 0 & $W_{2}^{\perp}$ & $W_{2}$ & $\mathbb{F}_{5}^{V}$ & $\mathbb{F}_{5}^{V}$ & $\mathbb{F}_{5}^{V}$ & $\mathbb{F}_{5}^{V}$ \\
\hline 1 & $\mathbb{F}_{5}^{V}$ & $W_{2}=R C_{0}+W_{1}$ & $\mathbb{F}_{5}^{V}$ & $W_{1} \oplus W_{2}^{\perp}$ & $\mathbb{F}_{5}^{V}$ & $W_{1} \oplus W_{2}^{\perp}$ \\
\hline 2 & $\mathbb{F}_{5}^{V}$ & $E\left(W_{2} \subseteq R C_{0} \subseteq W_{2}\right.$ & $\mathbb{F}_{5}^{V}$ & $\mathbb{F}_{5}^{V}$ & $E\left(C_{2}\right)=W_{2}^{\perp}+W_{1}$ & $\mathbb{F}_{5}^{V}$ \\
\hline 3 & $\langle\boldsymbol{\jmath}\rangle^{\perp}$ & $W_{2}$ & $\supseteq W_{2}^{\perp}$ & $\mathbb{F}_{5}^{V}$ & $\langle\boldsymbol{\jmath}\rangle^{\perp}$ & $\mathbb{F}_{5}^{V}$ \\
\hline 4 & $W_{1}^{\perp}$ & $W_{2}$ & $W_{1}^{\perp}$ & $\mathbb{F}_{5}^{V}$ & $\mathbb{F}_{5}^{V}$ & $E\left(W_{1}\right)^{\perp}$ \\
\hline \hline
\end{tabular}

Table $9 \Gamma(n, 3, r), R \Gamma(n, 3, r), p=5$, congruences modulo 5

\begin{tabular}{|l||l|l||l|l||l|l|}
\hline \hline$n$ cong & $C_{0}$ & $R C_{0}$ & $C_{1}$ & $R C_{1}$ & $C_{2}$ & $R C_{2}$ \\
\hline \hline 0 & $\mathbb{F}_{7}^{V}$ & $W_{2}$ & $\mathbb{F}_{7}^{V}$ & $\mathbb{F}_{7}^{V}$ & $W_{1} \oplus W_{2}^{\perp}$ & $\mathbb{F}_{7}^{V}$ \\
\hline 1 & $\mathbb{F}_{7}^{V}$ & $W_{2}$ & $\mathbb{F}_{7}^{V}$ & $\mathbb{F}_{7}^{V}$ & $W_{1}^{\perp}$ & $\mathbb{F}_{7}^{V}$ \\
\hline 2 & $\mathbb{F}_{7}^{V}$ & $E\left(W_{2}\right) \subseteq R C_{0} \subseteq W_{2}$ & $\supseteq W_{1}+W_{2}^{\perp}$ & $\mathbb{F}_{7}^{V}$ & $\mathbb{F}_{7}^{V}$ & $\mathbb{F}_{7}^{V}$ \\
\hline 3 & $\langle\boldsymbol{\jmath}\rangle^{\perp}$ & $W_{2}$ & $W_{1}^{\perp}$ & $\mathbb{F}_{7}^{V}$ & $\langle\boldsymbol{\jmath}\rangle^{\perp}$ & $\mathbb{F}_{7}^{V}$ \\
\hline 4 & $W_{1}^{\perp}$ & $W_{2}$ & $W_{1}^{\perp}$ & $\mathbb{F}_{7}^{V}$ & $\mathbb{F}_{7}^{V}$ & $E\left(W_{1}\right)^{\perp}$ \\
\hline 5 & $W_{2}^{\perp}$ & $W_{2}$ & $\mathbb{F}_{7}^{V}$ & $\mathbb{F}_{7}^{V}$ & $\langle\boldsymbol{\jmath}\rangle^{\perp}$ \\
\hline 6 & $\mathbb{F}_{7}^{V}$ & $W_{2}=R C_{0}+W_{1}$ & $\mathbb{F}_{7}^{V}$ & $W_{1} \oplus W_{2}^{\perp}$ & $\mathbb{F}_{7}^{V}$ & $W_{1} \oplus W_{2}^{\perp}$ \\
\hline \hline
\end{tabular}

Table $10 \Gamma(n, 3, r), R \Gamma(n, 3, r), p=7$, congruences modulo 7

Note that $\Gamma(n, 2,0)=K G_{n, 2}$, and $\Gamma(n, 2,1)=J(n, 2)$, the triangular graph. We summarize the results for $\Gamma(n, 2, r)$ and $R \Gamma(n, 2, r)$ for $p=2,3$ in Tables 11 and 12 , where $|V|=\left(\begin{array}{l}n \\ 2\end{array}\right)$. Some of the proofs can be found in $[14,8]$, and those that are not there can be deduced easily in a way similar to the proofs here for $k=3$. 


\begin{tabular}{||l||l|l|l|l||}
\hline \hline$n$ cong & $C_{0}$ & $R C_{0}$ & $C_{1}$ & $R C_{1}$ \\
\hline \hline 0 & $\mathbb{F}_{2}^{V}$ & $\left\langle E\left(W_{1}\right), \boldsymbol{\jmath}\right\rangle$ & $E\left(W_{1}\right)$ & $\mathbb{F}_{2}^{V}$ \\
\hline 1 & $W_{1}^{\perp}$ & $\left\langle W_{1}, \boldsymbol{\jmath}\right\rangle$ & $W_{1}$ & $W_{1}^{\perp}$ \\
\hline 2 & $\langle\boldsymbol{\jmath}\rangle^{\perp}$ & $\left\langle E\left(W_{1}\right), \boldsymbol{\jmath}\right\rangle$ & $E\left(W_{1}\right)$ & $\mathbb{F}_{2}^{V}$ \\
\hline 3 & $W_{\Pi}$ & $W_{\Pi}^{\perp}$ & $W_{1}$ & $W_{1}^{\perp}$ \\
\hline \hline
\end{tabular}

Table $11 \Gamma(n, 2, r), R \Gamma(n, 2, r), p=2$, congruences modulo 4

\begin{tabular}{||l||l|l|l|l||}
\hline \hline$n$ cong & $C_{0}$ & $R C_{0}$ & $C_{1}$ & $R C_{1}$ \\
\hline \hline 0 & $W_{\Pi}$ & $\mathbb{F}_{3}^{V}$ & $\mathbb{F}_{3}^{V}$ & $\left\langle W_{1}^{\perp}, \boldsymbol{\jmath}\right\rangle$ \\
\hline 1 & $\mathbb{F}_{3}^{V}$ & $E\left(W_{1}\right)^{\perp}$ & $E\left(W_{1}\right)^{\perp}$ & $\mathbb{F}_{3}^{V}$ \\
\hline 2 & $\langle\boldsymbol{\jmath}\rangle^{\perp}$ & $\mathbb{F}_{3}^{V}$ & $\langle\boldsymbol{\jmath}\rangle^{\perp}$ & $\mathbb{F}_{3}^{V}$ \\
\hline \hline
\end{tabular}

Table $12 \Gamma(n, 2, r), R \Gamma(n, 2, r), p=3$, congruences modulo 3

Thus, what was found for $k=2,3$, all $p$, and for other values of $k$ (for example for Johnson and odd graphs for all $k$ and $p=2$, from Fish [8]): the codes of $\Gamma(n, k, r)$ and $R \Gamma(n, k, r)$ are all some combination of the codes $C$, where $C$ is $W_{s}, E\left(W_{s}\right), W_{\Pi},\langle\boldsymbol{\jmath}\rangle, \mathbb{F}_{p}^{V}$, along with their duals and hulls. The tables do show some inconclusive results, but computational examination with Magma of these cases all indicate that the above statement holds in general.

We leave a more complete study of the codes $W_{i}, W_{\Pi}$, in the general case, to a future paper. We have also not included the codes from the complementary graphs in our discussion; these are uniform subset graphs only if $k=2$. Since our aim was to describe, as far as possible, the codes from $\Gamma(n, k, r)$ in terms of the $W_{i}, W_{\Pi}$, we have not listed the minimum weights in the tables, nor the hulls. Some of these can be deduced from Sections 3, 4, 5 and 7. In addition some of the codes are $L C D$, i.e. have zero hull, and this happens in particular when $C_{i}=R C_{i}^{\perp}$, since $C_{i}+R C_{i}=\mathbb{F}_{p}^{V}$. For example if $n \equiv 5(\bmod p), p \geq 5$, then from Tables 1 and $2, C_{0}^{\perp}=R C_{0}=W_{2}$. An example of a zero hull when $C \neq R C^{\perp}$ is from Tables 3 and 4 , where $C_{1}=W_{1}^{\perp}$ for $n \equiv 4(\bmod p)$, but $R C_{1} \neq W_{1}$, and from Corollary $2, \operatorname{Hull}\left(W_{1}\right)=\operatorname{Hull}\left(W_{1}^{\perp}\right)=\{0\}$. See also $[16]$ for further results on $L C D$ codes from graphs.

\section{References}

1. Araujo, J., Bratten, T.: On the spectrum of the Johnson graphs $J(n, k, r)$. In: Proceedings of the XXIIrd "Dr. Antonio A. R. Monteiro" Congress, pp. 57-62 (2016). Univ. Nac. Sur Dep. Mat. Inst. Mat., Baha Blanca, 2015

2. Assmus Jr, E.F., Key, J.D.: Designs and their Codes. Cambridge: Cambridge University Press (1992). Cambridge Tracts in Mathematics, Vol. 103 (Second printing with corrections, 1993)

3. Bosma, W., Cannon, J., Playoust, C.: The Magma algebra system I: The user language. J. Symbolic Comput. 24, 3/4, 235-265 (1997)

4. Brouwer, A., Haemers, W.: Association schemes. In: R. Graham, M. Grötschel, L. Lovász (eds.) Handbook of Combinatorics, pp. 749-771. Elsevier; MIT, Cambridge,MA (1995). Chapter 15, Vol. 1 
5. Cannon, J., Steel, A., White, G.: Linear codes over finite fields. In: J. Cannon, W. Bosma (eds.) Handbook of Magma Functions, pp. 3951-4023. Computational Algebra Group, Department of Mathematics, University of Sydney (2006). V2.13, http://magma.maths.usyd.edu.au/magma

6. Delsarte, P.: An algebraic approach to the association schemes of coding theory. Tech. rep., Philips Research Laboratorie (1973). Philips Research Reports, Supplement No. 10

7. Delsarte, P., Levenshtein, V.I.: Association schemes and coding theory. IEEE Trans. Inform. Theory 44, 2477-2504 (1998)

8. Fish, W.: Codes from uniform subset graphs and cycle products. Ph.D. thesis, University of the Western Cape (2007)

9. Fish, W.: Binary codes and partial permutation decoding sets from the Johnson graphs. Graphs Combin. 31, 1381-1396 (2015)

10. Fish, W., Fray, R., Mwambene, E.: Binary codes and partial permutation decoding sets from the odd graphs. Cent. Eur. J. Math. 12 (9), 1362 -1371 (2014)

11. Fish, W., Key, J.D., Mwambene, E.: Ternary codes from reflexive graphs on 3-sets. Appl. Algebra Engrg. Comm. Comput. 25, 363-382 (2014)

12. Fish, W., Key, J.D., Mwambene, E.: Binary codes from reflexive graphs on 3-sets. Adv. Math. Commun. 9, 211-232 (2015)

13. Huffman, W.C.: Codes and groups. In: V.S. Pless, W.C. Huffman (eds.) Handbook of Coding Theory, pp. 1345-1440. Amsterdam: Elsevier (1998). Volume 2, Part 2, Chapter 17

14. Key, J.D., Moori, J., Rodrigues, B.G.: Binary codes from graphs on triples. Discrete Math. 282/1-3, 171-182 (2004)

15. Key, J.D., Moori, J., Rodrigues, B.G.: Ternary codes from graphs on triples. Discrete Math. 309, 4663-4681 (2009)

16. Key, J.D., Rodrigues, B.G.: $L C D$ codes from adjacency matrices of graphs. (To appear) Appl. Algebra Engrg. Comm. Comput. DOI: 10.1007/s00200-017-0339-6

17. Kirkman, T.P.: On a problem in combinations. Cambridge and Dublin Math. J. 2, 191-204 (1847)

18. Krebs, M., Shaheen, A.: On the spectra of Johnson graphs. Electron. J. Linear Algebra $\mathbf{1 7}, 154-167(2008)$

19. Massey, J.L.: Linear codes with complementary duals. Discrete Math. 106/107, 337$342(1992)$

20. Moore, E.H.: Concerning triple systems. Math. Ann. 43, 271-285 (1893)

21. Peeters, R.: On the $p$-ranks of the adjacency matrices of distance-regular graphs. J. Algebraic Combin. 15, 127-149 (2002) 